\title{
An Observing System Simulation Experiment for the Impact of MTG Candidate Infrared Sounding Mission on Regional Forecasts: System Development and Preliminary Results
}

\author{
Hongli Wang, ${ }^{1}$ Xiang-Yu Huang, ${ }^{1}$ and Yongsheng Chen ${ }^{1,2}$ \\ ${ }^{1}$ National Center for Atmospheric Research, P.O. Box 3000, Boulder, CO 80307, USA \\ ${ }^{2}$ York University, Toronto, ON, Canada M3J 1 P3 \\ Correspondence should be addressed to Hongli Wang; hlwang@ucar.edu
}

Received 11 February 2013; Accepted 1 March 2013

Academic Editors: R. Fraile, T. Georgiadis, K. Nakamura, and Z. Pu

Copyright (C) 2013 Hongli Wang et al. This is an open access article distributed under the Creative Commons Attribution License, which permits unrestricted use, distribution, and reproduction in any medium, provided the original work is properly cited.

An Observing System Simulation Experiment (OSSE) was designed and developed to assess the potential benefit of the Infrared Sounding on the Meteosat Third Generation (MTG-IRS) geostationary meteorological satellite system to regional forecasts. In the proposed OSSE framework, two different models, namely, the MM5 and WRF models, were used in a nature run and data assimilation experiments, respectively, to reduce the identical twin problem. The 5-day nature run, which included three convective storms that occurred during the period from 11 to 16 June 2002 over US Great Plains, was generated using MM5 with a $4 \mathrm{~km}$. The simulated "conventional" observations and MTG-IRS retrieved temperature and humidity profiles, produced from the nature run, were then assimilated into the WRF model. Calibration experiments showed that assimilating real or simulated "conventional" observations yielded similar error statistics in analyses and forecasts, indicating that the developed OSSE system worked well. On average, the MTG-IRS retrieved profiles had positive impact on the analyses and forecasts. The analyses reduced the errors not only in the temperature and the humidity fields but in the horizontal wind fields as well. The forecast skills of these variables were improved up to 12 hours. The $18 \mathrm{~h}$ precipitation forecast accuracy was also increased.

\section{Introduction}

Remotely sensed satellite observations play an important role in modern data assimilation and forecast systems $[1,2]$. Satellite observations in the visible, infrared, and microwave spectrum provide a wealth of information on the atmosphere states. The variational data assimilation technique has been pursued in research communities and operational centres, with the main focus on large scale and mesoscale forecast, to assimilate clear sky and cloudy radiance. For example, at the European Centre for Medium-Range Weather Forecasts (ECMWF), an all-sky approach [3] has been developed to assimilate Special Sensor Microwave/Imager (SSM/I) and Advanced Microwave Scanning Radiometer for the Earth Observing system (AMSR-E) data. At National Oceanic and Atmospheric Administration (NOAA), Weng et al. [4] developed a hybrid variational scheme to use observations from the Advanced Microwave Sounding Unit (AMSU) instruments. Until recently, more attention has been directed towards cloud-resolving scale data assimilation. Cloudy infrared radiance assimilation at convection-resolving scale using a 4-dimensional variational data assimilation system was studied by Vukicevic et al. [5,6]. Overall their results indicate that the analyzed atmospheric fields are improved after assimilation.

The European Organisation for the Exploitation of Meteorological Satellites (EUMETSAT) is preparing for the next European operational geostationary meteorological satellite system named the Meteosat Third Generation (MTG). The MTG series will comprise six satellites, with the first spacecraft likely to be ready for launch in 2017. This program will provide space-acquired meteorological data until at least the late 2030s. Details can be found at the EUMETSAT website (http://www.eumetsat.int). Compared to the Meteosat Second Generation (MSG), the inclusion of an Infrared Sounding on the MTG (MTG-IRS) will be a new capability, 
which will support regional and convective-scale numerical weather prediction in Europe, through unprecedented detail on wind, temperature, and humidity, at high vertical, horizontal, and temporal resolution [7].

Assimilating the MTG-IRS observations into a mesoscale model is expected to improve regional numerical weather forecast skills. Recent studies suggest that the accurate representation of the low-level water vapor and temperature is crucial for the quantitative precipitation forecast $[8,9]$. When realistic mesoscale details of the horizontal variations in moisture and temperature are included, pronounced improvements in forecast skills for convective events can be achieved [10-13]. To evaluate such potentials, quantitative analyses of the added values of the IRS candidate mission for regional scale forecasts are performed by the means of the Observing System Simulation Experiment (OSSE).

The OSSE method is regarded as an efficient way to test the impact of future observing systems and new data assimilation techniques on weather analysis and prediction [14-17]. A typical OSSE system includes the following components: a nature run or "truth" run, simulated observations, and data assimilation and forecast experiment to evaluate the impact of simulated observations. The calibration of OSSE assesses the performance of OSSE by comparing the data impact of existing instruments to their simulated data impact [14]. The OSSE should reproduce the same/similar real data impact.

The OSSE system may overestimate benefit of the observations when the same numerical weather model is used in the nature run and forecast experiment (i.e., the identical twin problem). To alleviate this problem, a model with different physics schemes is often used in regional OSSE to simulate model errors [16]. In this paper, we employed two different models in nature run and data assimilation experiments to further reduce the identical twin problem in the regional OSSE. The Pennsylvania State University/National Center for Atmospheric Research (NCAR) Mesoscale Model Version 5 (MM5) was utilized to produce the nature run, whereas the Weather Research and Forecasting (WRF) model was used as the forecast model. The MM5 model and WRF model have different numerical solvers and different physics parameterizations and grid sizes. Analyses (forecasts) from different datasets are employed to initialize initial and boundary conditions. It is expected that differences between two models can reasonably represent the model error. A pair of calibration experiments, that is, one assimilates real observations while the other assimilates simulated observations, were conducted to demonstrate the performance of the developed OSSE system.

This paper describes the framework design of the OSSE system and summarizes the preliminary results of the potential benefit of MTG-IRS on regional forecasts of three convective storms occurred over the US Great Plains in June 2002. The OSSE system is characterized by a nature run using the MM5 model with convective system resolving grid size of $4 \mathrm{~km}$, (rapid update) cycling data assimilation and forecast experiments using the WRF model with $12 \mathrm{~km}$ resolution, and a pair of calibration experiments. The overview of selected cases is in Section 2. The methodology and experiment setup are described in Section 3. Section 4 presents the results of the OSSE. A summary and discussion are given in Section 5.

\section{Overview of Selected Cases}

Three convection cases that occurred between 11 and 16 June 2002 during the International $\mathrm{H}_{2} \mathrm{O}$ Project (IHOP 2002) are selected. These three cases include dry line, convective storms, and a severe mesoscale convective system (MCS). On 11 June 2002, a dry line formed in the Oklahoma panhandle in the late afternoon. Three storms developed on this particular day in regions close to the border of Colorado and Oklahoma. On 12 June 2002, a northeast-to-southwest-oriented squall line from the Kansas and Oklahoma border to the Texas Panhandle was initiated at around 2100 UTC 12 June 2002. At the same time, isolated convections formed from the Kansas and Oklahoma border to Texas. They gradually strengthened along most parts of the dry line, and a severe storm developed near a triple point that was formed by the dry line and a cold air outflow. The 15-16 June case is a severe MCS that occurred over the US central and southern plains in the late afternoon 15 June 2002. The MCS produced severe weather including several tornadoes in southern Kansas, and a swath of wind damage reports through central Oklahoma southward through central Texas as it propagated south-southeastward. More detailed descriptions of three cases were reported by Wechwerth et al. [13], Dawson and Xue [18], and Wang et al. [19].

\section{Methodology and Experiment Design}

3.1. Observing System Simulation Experiment. The OSSE flowchart is illustrated in Figure 1. The nature run (or "truth" run) serves as a true atmosphere for the OSSE. The observational data for existing and testing (nonexiting) instruments are simulated from the "truth." The added values of the testing instruments (MTG-IRS) are evaluated by cycling data assimilation and forecast experiments with and without simulated MTG-IRS observations. In addition to evaluating the impact of testing instruments, another benefit of OSSE is that the "truth" is known and forecast skills can be assessed easily. In this study, a calibration run by performing a pair of experiments, that is, assimilating real or simulated observations is conducted to demonstrate the performance of the developed OSSE system. Although it may be difficult to reproduce the exact real data impact in the OSSE, it should reproduce the similar real data impact.

In previous published OSSE studies, for example, Masutani et al. [14] used two different global models at ECMWF and NCEP to mimic model error; and Chen et al. [16] used the same WRF model but different physics schemes in nature run and data assimilation experiment to simulate model errors. In this study, the nature run, data assimilation, and forecast experiments employ two different mesoscale models to simulate model errors and thus reduce the identical twin problem. To further reduce the identical twin problem, two models were initialized from two different datasets. The Eta model analyses at $40 \mathrm{~km}$ resolution were used to generate 


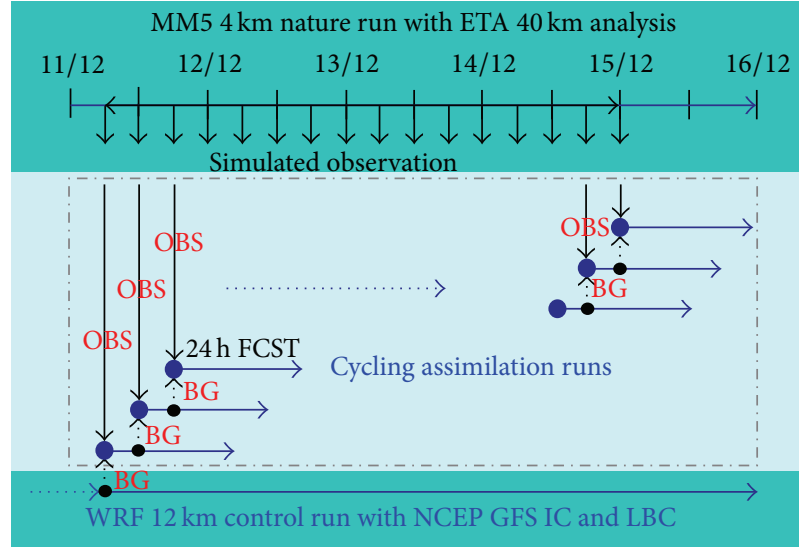

FIGURE 1: Flowchart of experiments.

initial and boundary conditions for the nature run, while the National Center for Environment Prediction (NCEP) Global Forecast System (GFS) analyses and forecasts reduced to $1^{\circ} \times 1^{\circ}$ resolution were used to generate initial and boundary conditions for WRF model in the data assimilation experiments. Though it is not easy to verify how realistic the model error is since we do not know much on "true model error," the above steps are expected to simulate model error in realworld data assimilation.

3.2. Models and Data Assimilation System. The MM5 model version 3.6 [20] and the WRF model [21] are employed in the nature run and data assimilation experiments, respectively. MM5 is a limited-area, nonhydrostatic, and terrain-following sigma-coordinate model, which is designed to simulate mesoscale atmospheric circulation. MM5 uses leapfrog step time differencing with an Asselin filter. The WRF model produces the background for the data assimilation experiments and makes forecasts from the analyses. The WRF model is a nonhydrostatic, terrain-following mass vertical coordinate numerical weather prediction and atmospheric simulation system. The model uses a third-order Runge-Kutta time integration, the third-to-fifth-order advection operators, and split-explicit fast wave integration scheme conserving both mass and energy.

The data assimilation system is the WRF Variational Data Assimilation System (WRF-Var) [22, 23]. The WRF-Var developed at NCAR is a unified (global/regional, multimodel, 3/4D-Var) model-space variational data assimilation system. A wide range of observation types, including conventional observations, radar, and satellite radiances, can be assimilated in WRF-Var. The 3-dimensional component of WRF-Var (WRF 3D-Var) is employed in this study. A new observation type named MTG-IRS is added into WRF 3D-Var to assimilate retrieved MTG-IRS temperature and water vapor mixing ratio profiles directly.

\subsection{Experiment Setup}

3.3.1. Nature Run. The model configuration for the nature run in this study employs $505 \times 505$ grid points with $4 \mathrm{~km}$ horizontal resolution and 35 vertical levels. The model top is at $50 \mathrm{hPa}$. The domain covers the central areas of the United States (Figure 2). The Medium Range Forecast boundary layer scheme [24] and the Reisener microphysics scheme [25] are used. No cumulus parameterization scheme is used. The MM5 model is initialized at 1200 UTC 11 June 2002 and runs for 5 days. The initial and boundary conditions are interpolated from the NCEP Eta model $40 \mathrm{~km}$ analyses. It is noted that no data assimilation is applied in the nature run. The model state is saved hourly and then used as the "true" atmospheric state from which the observations will be simulated.

3.3.2. Simulated Observations. Given the true state, simulated observations can be generated. Conventional observations such as radiosonde, pibal, surface station observations, and aircraft report are simulated to provide the basic simulated observing system for the reference data assimilation experiment. The satellite wind observations are generated as well. For simplicity, the term "conventional" observation is defined to include all the observation types (e.g., satellite wind) listed above. The types and positions of the real observations are obtained from the NCEP Automated Data Processing observations. Readers are referred to see websites http://rda.ucar .edu/ and http://rda.ucar.edu/datasets/ds464.0/ for detailed descriptions of the data. The forward observation operators of WRF-Var are conveniently employed to produce simulated conventional observations using the atmospheric state in the MM5 nature run. First, the true simulated observations are obtained, and the realistic observation errors with Gaussian distributions are then added. The default observation error statistics in WRF-Var system are used. For specific values, readers should refer to the observation error file (obserr.txt) in WRF-Var software (http://www.mmm.ucar .ucar.edu/wrf/users/wrfda/).

Simulated MTG-IRS temperature and moisture profiles are generated hourly from the nature run. The package to perform these simulated retrievals has been used for the Geostationary Operational Environmental Satellite-R Series (GOES-R) Hyperspectral Environmental Suite (HES) tradeoff studies at Cooperative Institute for Meteorological Satellite Studies (CIMSS) and described in various publications (e.g., [28]). The retrieval package consists of two components: (1) simulation of the MTG-IRS observations and (2) Principle Component Regression (PCR) retrieval.

(i) Simulation of the MTG-IRS Observations. According to the Meteosat Third Generation Mission Requirement Document (MRD), the MTG-IRS candidate mission shall make observations in two broad spectral bands, namely, a LWIR band from $700-1210 \mathrm{~cm}^{-1}$ and a MWIR band from $1600-2175 \mathrm{~cm}^{-1}$. The radiometric noise will range from about 0.2 to $0.9 \mathrm{~K}$. A fast and accurate transmittance models called Pressure layer Fast Algorithm for Atmospheric Transmittances (PFAAST) [26] is chosen to simulate clear sky transmission profiles. This model was used to simulate observations for the Advanced Baseline Imager (ABI)/HES candidate missions [27]. The calculations are made at 101 pressure levels $(0.1-1050 \mathrm{hPa})$. 


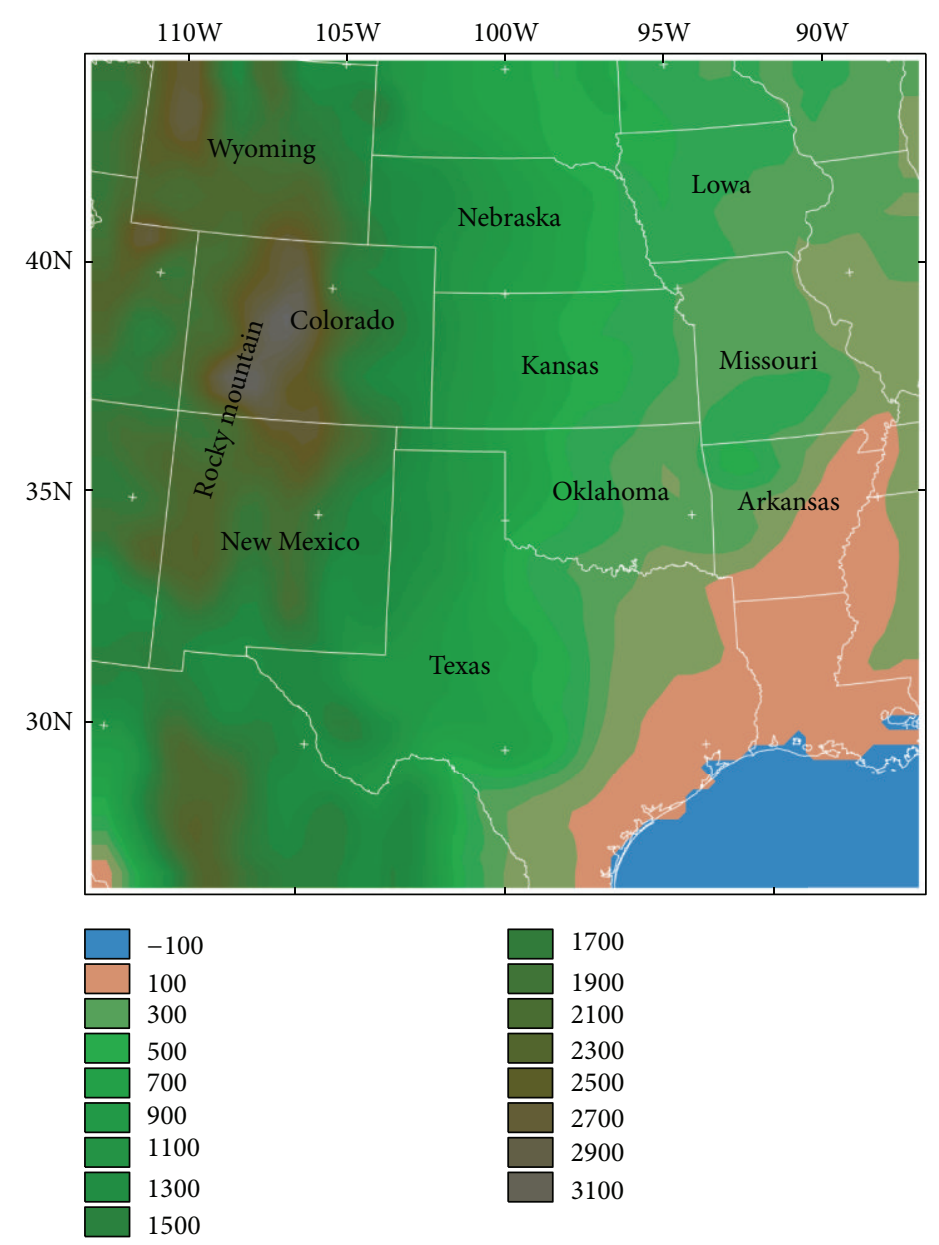

FIGURE 2: Model domain superposed with topography (unit: m) for both MM5 and WRF. The states and Rocky mountains are also indicated.

The output from nature run is interpolated into these 101 pressure levels and used as input to the transmittance model. The temperature and humidity retrievals are performed on these 101 pressure levels.

(ii) PCR Retrieval. Once the radiances at top of atmosphere in the MTG-IRS channels for a set of atmospheric conditions have been calculated, they are inverted to get simulated retrieval profiles of temperature, moisture, and the surface skin temperature. The derivation of the regression coefficients is done on a dataset extracted from the nature run. As with all statistical regression methods, the accuracy of the retrievals depends upon the adopted training dataset. A training dataset was sampled from the nature run every 6 hours such that a representative dataset was obtained. This means that the training dataset is not completely independent from the dataset used for the retrievals. However, it is important to realize that the PCR retrieval is the first step in the complete retrieval process. After the PCR retrieval, a nonlinear iterative physical retrieval on the synthetic observations should be performed. The objective of the PCR retrieval is therefore to provide a starting point for the nonlinear iterative physical retrieval, which is close to the final solution to reduce the number of iterations.
The radiance dataset calculated for the training dataset was used to calculate empirical orthogonal functions (EOF) using a standard singular value decomposition of the covariance matrix. Values for the radiometric noise are added in the generation of the regression coefficients from a correlation of the EOFs with the training dataset. The actual PCR retrieval is applied to the simulated MTG-IRS radiances. First these radiances are projected onto the EOFs derived from the training dataset. Values for temperature, moisture, and surface temperature are then obtained after the application of the regression relations. After the PCR retrieval, a nonlinear iterative procedure is applied to the radiative transfer equation to further improve the profiles [28].

Currently, the MTG-IRS retrieval algorithm can only provide temperature and humidity profiles over the clear sky regions. In addition, the retrieval algorithm cannot deal with complicated land surfaces over the Rocky Mountains. Thus, there are no retrievals over the Rocky Mountain. But in other clear sky regions in the model domain, the retrieval algorithm worked well.

3.3.3. Data Assimilation and Forecast Experiment. The MTGIRS retrieved profiles are assimilated in the presence of other simulated conventional observations to assess their added 
TABLE 1: List of experiments.

\begin{tabular}{|c|c|c|c|}
\hline Experiment & Cycling period & Resolution (km) & Initial condition and assimilated data \\
\hline \multicolumn{4}{|l|}{ NoDA } \\
\hline Nature & No & 4 & ETA $40 \mathrm{~km}$ analysis \\
\hline Control & No & 12 & GFS analysis and forecast, no observations \\
\hline \multicolumn{4}{|l|}{ 3DVar } \\
\hline ROP & $6 \mathrm{~h}$ & 12 & Background $(\mathrm{BG})+$ real conventional observations \\
\hline SOP-6hc & $6 \mathrm{~h}$ & 12 & $\mathrm{BG}+$ simulated conventional observations (SOP) \\
\hline SOP-RPtq-6hc & $6 \mathrm{~h}$ & 12 & $\mathrm{BG}+\mathrm{SOP}+$ retrieved profiles $(\mathrm{RP})$ of temperature $T$ and humidity $q(\mathrm{RPtq})$ \\
\hline SOP-RPq-6hc & $6 \mathrm{~h}$ & 12 & $\mathrm{BG}+\mathrm{SOP}+$ retrieved profiles of humidity $(\mathrm{RPq})$ \\
\hline SOP-1hc & $1 \mathrm{~h}$ & 12 & $\mathrm{BG}+\mathrm{SOP}$ \\
\hline SOP-RPtq-1hc & $1 \mathrm{~h}$ & 12 & $\mathrm{BG}+\mathrm{SOP}+\mathrm{RPtq}$ \\
\hline POP & $6 \mathrm{~h}$ & 12 & $\mathrm{BG}+$ perfect observation $(\mathrm{POP})$ \\
\hline
\end{tabular}

values. The experiments are listed in Table 1 and illustrated in Figure 1. These experiments shall provide reasonable assessments of the added value of the MTG-IRS retrievals.

The simulated data with high spatial density are thinned before data assimilation. Satellite winds are thinned to $36 \mathrm{~km}$ grids, and their errors are then assumed to be independent. The MTG-IRS retrievals have high horizontal error correlations, and they are thinned to $36 \mathrm{~km}$ grids for data assimilation. Vertical correlation in retrievals is not considered in data assimilation. However, retrievals are thinned to the 35 vertical pressure levels in WRF model.

Note that in all the control and assimilation experiments, the initial and boundary conditions of the WRF model are interpolated from the 1-degree resolution NCEP GFS analyses and subsequent 5-day forecasts at 0000 UTC 11 June 2002. The physics schemes chosen in the WRF simulations include the Noah land surface model, the WSM6 microphysics scheme [29] and the Grell scheme [30] for cumulus parameterization. The WRF model domain covers the same area as MM5. Limited by the available computational resources, all the experiments are conducted at $12 \mathrm{~km}$ resolution. We conducted several data assimilation and forecast experiments at $4 \mathrm{~km}$ at $18 \mathrm{Z}$ to show the sensitivity to model resolutions. We found that high-resolution simulations only slightly increase precipitation forecast skill. We also found that synoptic forcing plays an important role in this case. That is perhaps the reason that the above experiments did not show significant sensitivity to resolutions. It is reasonable to believe that the general conclusions drawn here also apply to $4 \mathrm{~km}$ resolution.

The background error covariance is generated by the National Meteorological Center (NMC) method [31]. Specifically, a set of cold-start forecasts is initialized from the NCEP GFS analyses and subsequent forecasts at 0000 UTC and 1200 UTC every day from 5 June 2002 to 19 June 2002. The differences of $24 \mathrm{~h}$ and $12 \mathrm{~h}$ forecasts are used to derive the background error covariance.

The control run is performed without data assimilation. In the control experiment, the WRF model is initialized from the GFS analysis at 0000 UTC 11 June 2002 and integrated for 5.5 days. Its $18 \mathrm{~h}$ forecast valid at 1800 UTC 11 June 2002 serves as the background (BG) fields for the first cycle of other data assimilation experiments. The control run also serves as the benchmark for intercomparison with other experiments with observations assimilated.

The added value of MTG-IRS retrievals is assessed in a cycling data assimilation and forecast mode. In a cycling mode, a previous forecast is used as the first guess for the current analysis. The data assimilation experiments, SOP-6hc and SOP-1hc, in which only simulated conventional observations are assimilated, are references experiment for quantifying MTG-IRS data impact. To validate the performance of the designed OSSE, we also conducted an experiment, ROP, in which the real conventional observations are assimilated. In OSSE, the assimilation of real observations will yield similar error statistics in analyses and forecasts compared to the assimilation of corresponding simulated observations.

To assess the added value of the retrieved MTG-IRS temperature and moisture profiles, the simulated conventional observations are assimilated in all data assimilation experiments. For example, in the experiment SOP-RPq-6hc, only the MTG-IRS humidity profiles are assimilated in addition to the simulated conventional data every 6 hours, while the experiment SOP-RPtq-6hc uses both MTG-IRS temperature and humidity profiles. The experiment SOP-RPtq-1hc is the same as SOP-RPtq-6hc except the assimilation cycling period is 1 hour instead of 6 hours.

The POP experiment is designed to assess the maximum impact of the perfect observations. The temperature, moisture, and wind profiles obtained directly from the truth at every $36 \mathrm{~km} \times 36 \mathrm{~km}$ grid point are assimilated in this experiment.

3.3.4. Verification. To objectively evaluate the impacts of MTG-IRS data on the regional scale analysis and forecast, traditional skill scores such as the root-mean-square (RMS) error between an experiment and the "truth" are computed.

The impact on precipitation forecast is quantified in terms of Equitable Threat Score (ETS) and frequency bias. The ETS is defined as

$$
\text { ETS }=\frac{\left(\text { hits }- \text { hits }_{\text {random }}\right)}{\left(\text { hits }+ \text { misses }+ \text { false alarms }- \text { hits }_{\text {random }}\right)},
$$

where

$$
\text { hits }_{\text {random }}=\frac{(\text { hits }+ \text { misses })(\text { hits }+ \text { false alarms })}{\text { Nobs }} .
$$




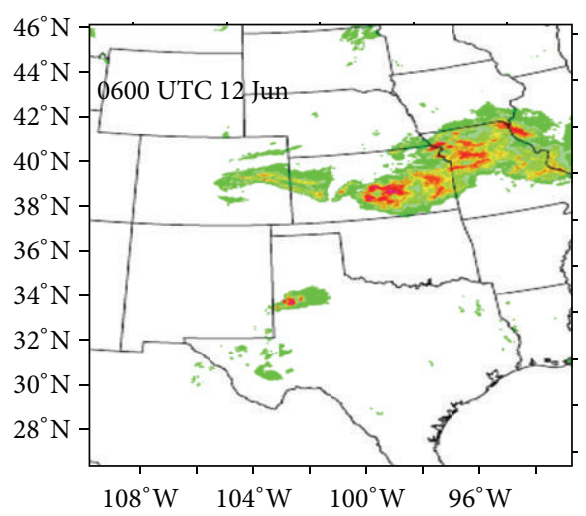

(a)

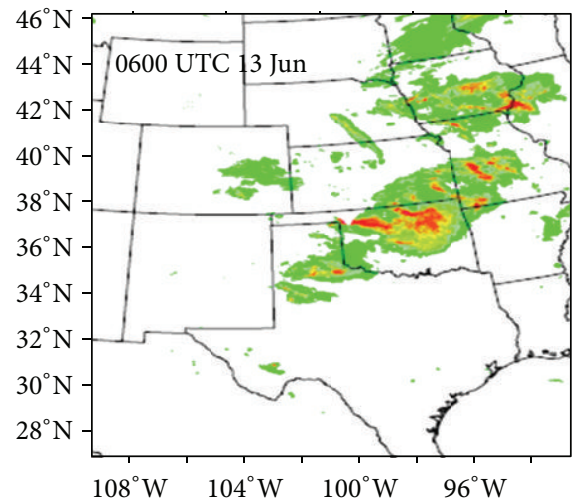

(c)
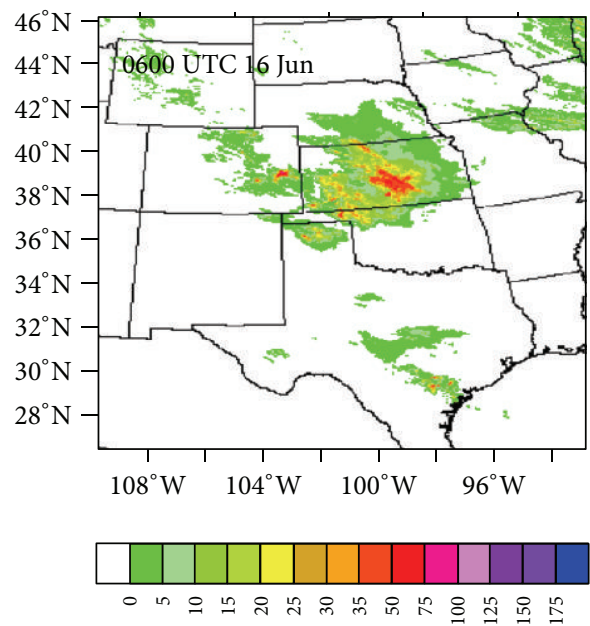

(e)

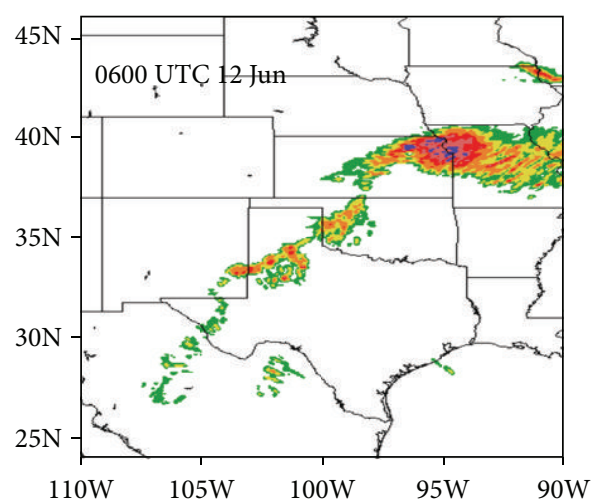

(b)

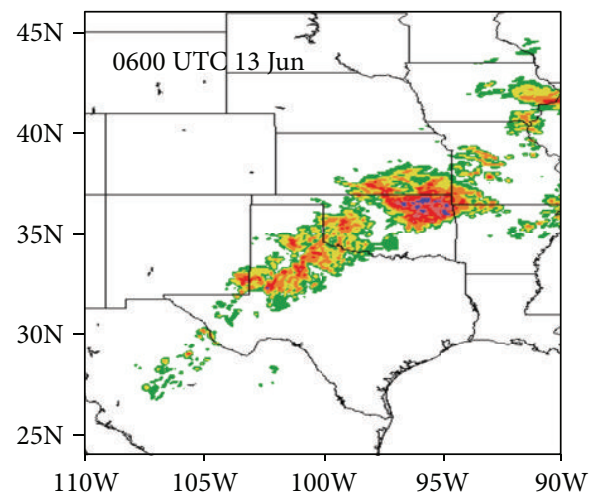

(d)

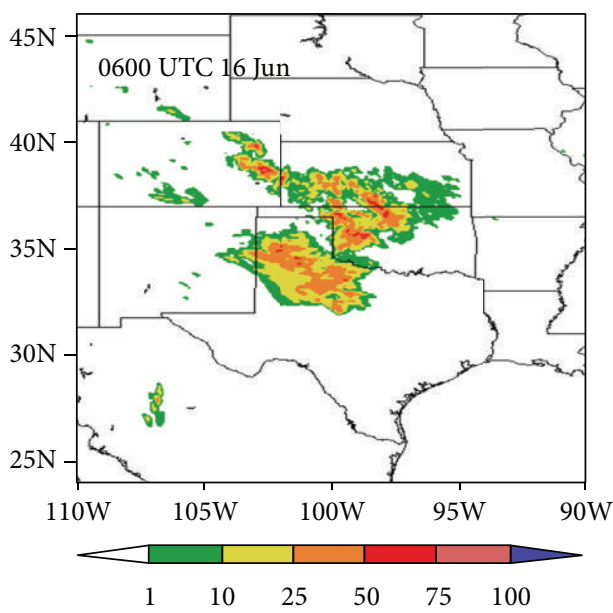

(f)

FIGURE 3: $6 \mathrm{~h}$ accumulated precipitation in the observation (a), (c), and (e) and in the nature run (b), (d), and (f) valid at 0600 UTC 12 (a) and (b), 0600 UTC 13 (c) and (d) and 0600 UTC 16 (e) and (f) June 2002. Note that, the color scales and map projections are different between the observation and the simulation.

The frequency bias is the ratio of the forecast frequency to the observed frequency, that is,

$$
\text { frequency bias }=\frac{\text { (hits }+ \text { false alarms })}{\text { (hits }+ \text { misses })} \text {. }
$$

The units of "hits," "misses," and "false alarms" are the number of grid points where the precipitation exceeds a specified threshold. Nobs are the total number of grid points being evaluated. If a forecast is perfect, ETS and frequency bias are 1.0 .

\section{Results}

4.1. The Nature Run. A 5-day nature run covering the selected three IHOP 2002 convective events is completed first. Figure 3 compares the $6 \mathrm{~h}$ accumulated precipitation of 


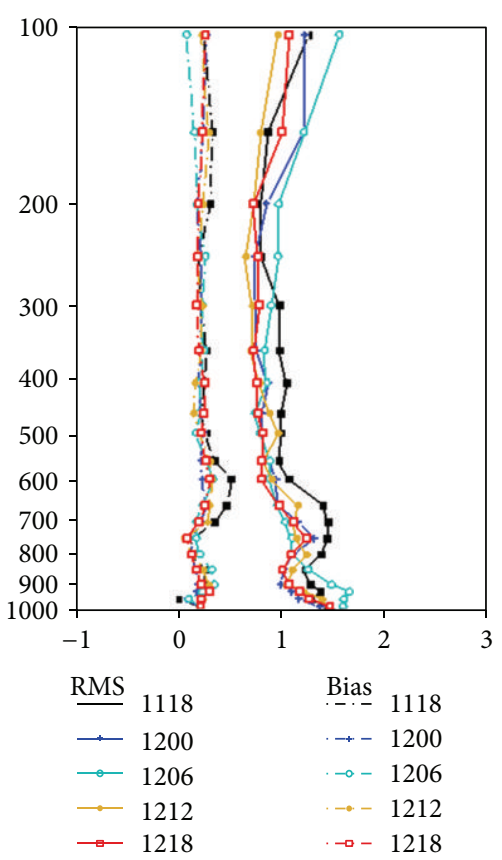

(a)

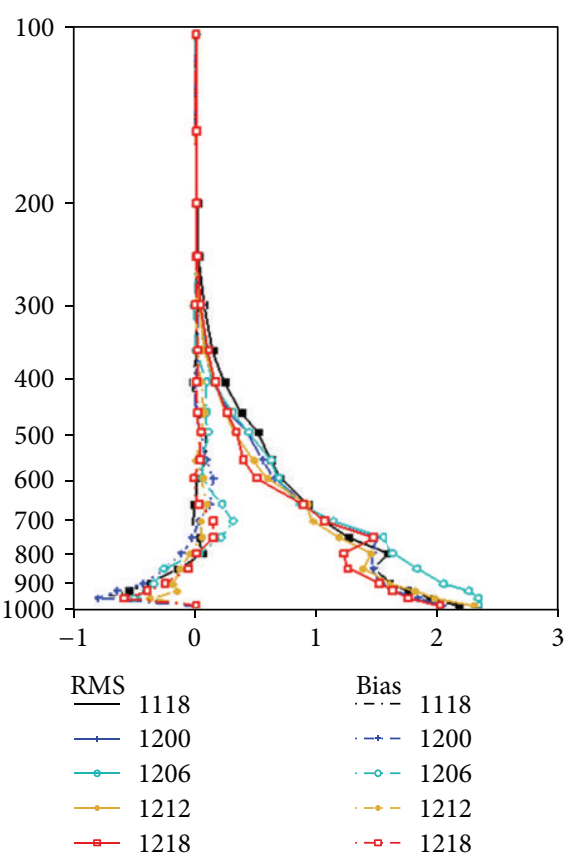

(b)

FIGURE 4: Bias and RMS error of (a) the retrieved profiles of $T(\mathrm{~K})$ and (b) water vapor mixing ratio $\left(\mathrm{g} \mathrm{kg}^{-1}\right)$ valid at different times. Legends show day and hour.

the nature run to the observations. The rainfall observations used here are the Stage IV analyses, which are based on the multisensor hourly/6-hourly "Stage III" analyses on local $4 \mathrm{~km}$ polar-stereographic grids produced by the 12 River Forecast Centers. For more description, the reader is referred to http://data.eol.ucar.edu/codiac/dss/id=21.093. The nature run slightly over-estimates the precipitation for the first case and underestimates the precipitation for the third case. In general, the MM5 nature run simulates well the positions of the three rainfall events. Comparing nature run with the NCEP Eta model $40 \mathrm{~km}$ analyses, it is found that nature run also gives a good simulation of large scale and mesoscale weather systems. Thus, the simulated atmospheric state from nature run is serviced as the "true" atmosphere.

\subsection{Simulated Observations}

4.2.1. Conventional Observations. The numbers of upper air observations including radiosondes and pibals vary from cycle to cycle, more at 0000 UTC and 1200 UTC and less at 0600 UTC and 1800 UTC. The upper air observations at 0000 UTC and 1200 UTC are about 30. For surface observations, the numbers remain almost unchanged throughout the $6 \mathrm{~h}$ cycles. The typical number of surface observation is about 650 . The numbers and positions of the satellite wind and MTG-IRS retrievals can change dramatically due to weather conditions.

4.2.2. MTG-IRS Retrievals. The temperature and water vapor mixing ratio profiles over clear air regions are retrieved. By comparing the retrievals with the "truth," it is found that the retrievals faithfully represent the relatively large-scale patterns and some mesoscale details of the real temperature field, especially in the middle atmosphere (figures not shown). The bias and RMS errors are shown in Figure 4. The temperature retrievals are more accurate in the middle levels from 600 to $200 \mathrm{hPa}$. The RMS error is close or smaller than $1 \mathrm{~K}$, which is comparable to the Atmospheric Infrared Sounder (AIRS) and the Infrared Atmospheric Sounding Interferometer (IASI) retrievals [32, 33]. For humidity, the retrieved water vapor mixing ratio has maximum RMS errors near the surface, and dry bias below $800 \mathrm{hPa}$. The maximum error is about $2 \mathrm{~g} \mathrm{~kg}^{-1}$, which is a little bigger than that from IASI retrieval [33]. Though it may not be straightforward to compare the MTG-IRS retrievals with the AIRS and IASI retrievals, we think that errors in MTG-IRS retrievals are reasonable and acceptable.

4.3. Calibration Experiments. To validate the performance of the designed OSSE, we first conducted a pair of calibration experiments ROP and SOP-6hc to compare the impacts from assimilating real conventional observations to assimilating corresponding simulated observations. If the designed OSSE system works well, the assimilation of real observations will yield similar error statistics in analyses and forecasts compared to the assimilation of simulated observations. Figure 5 shows the bias and RMS errors for temperature, water vapor mixing ratio, and $u$-wind and $v$-wind component of analyses and forecasts. Two sets of observations produce similar error statistics. It indicates that the developed OSSE system performs reasonably well.

4.4. 6-Hourly Cycling Experiments. The WRF 3D-Var analyses were performed in cycling mode starting from 1800 UTC 


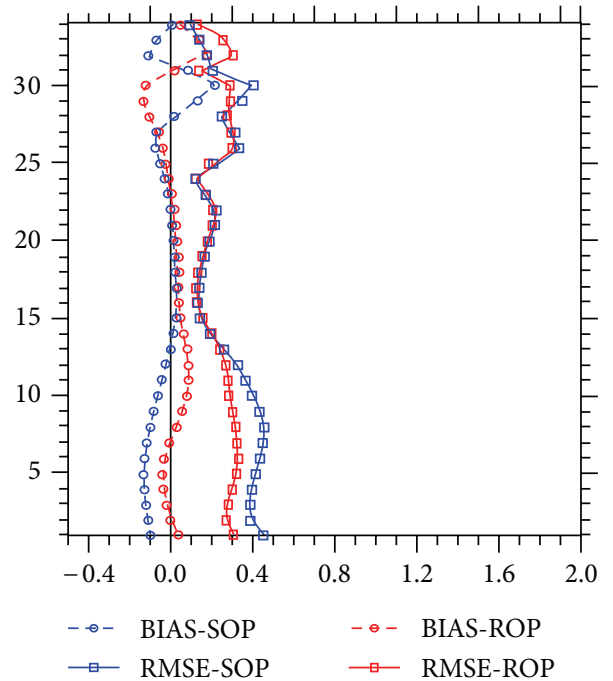

(a)

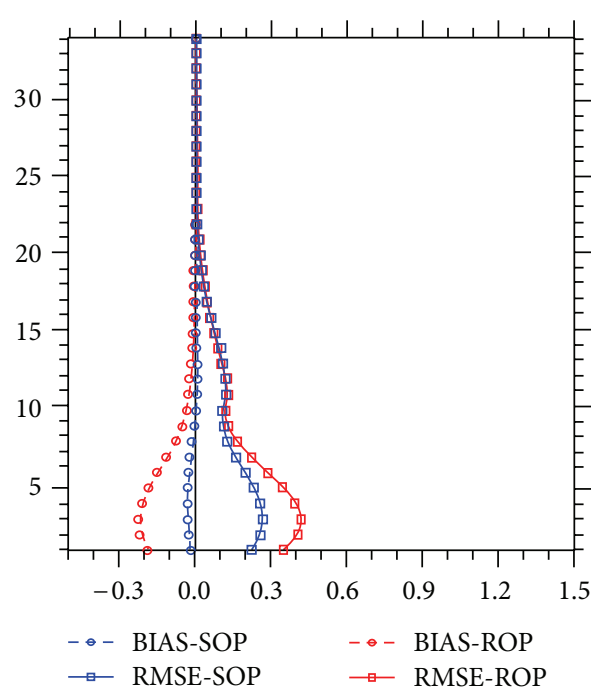

(c)

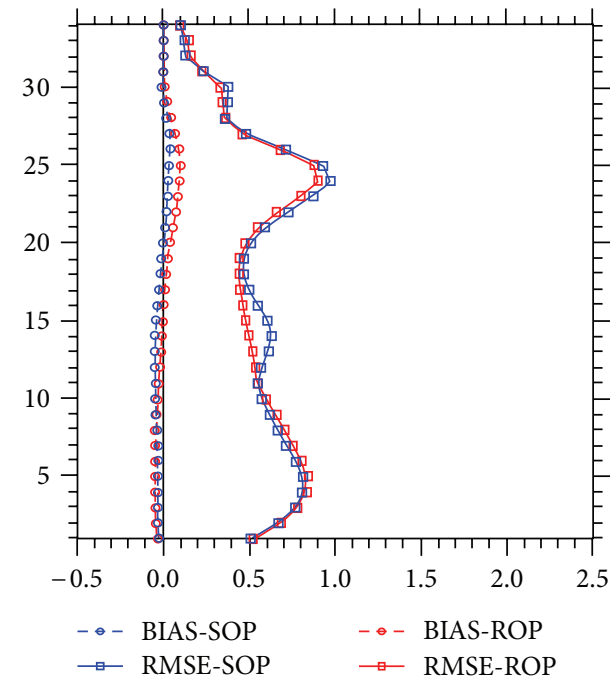

(e)

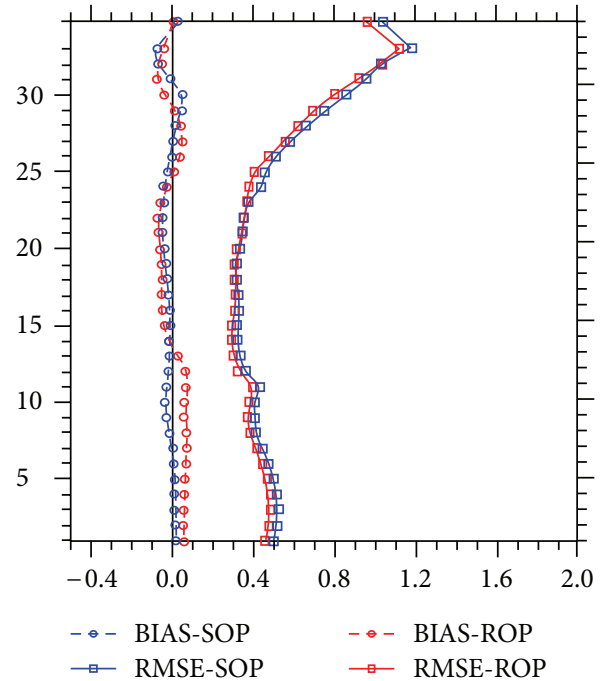

(b)

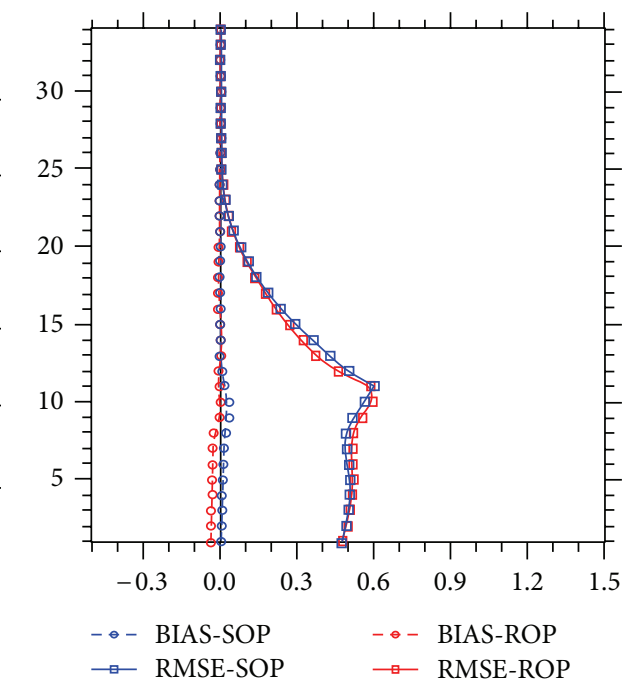

(d)

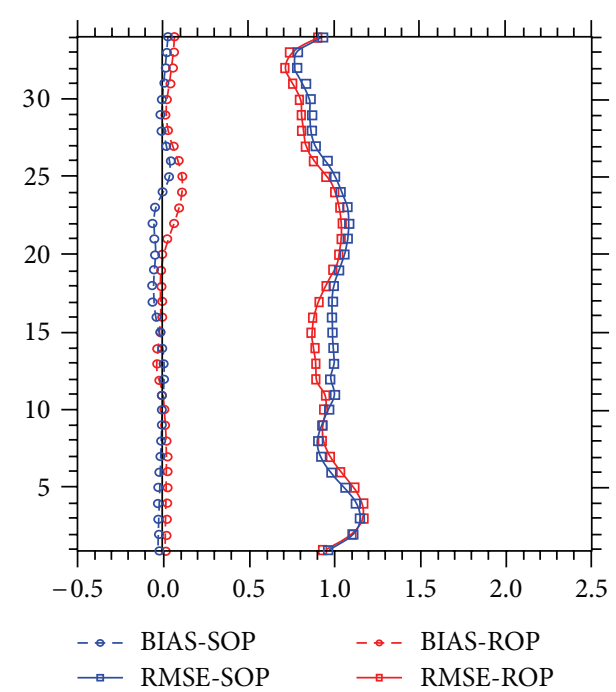

(f)

FIgUre 5: Continued. 


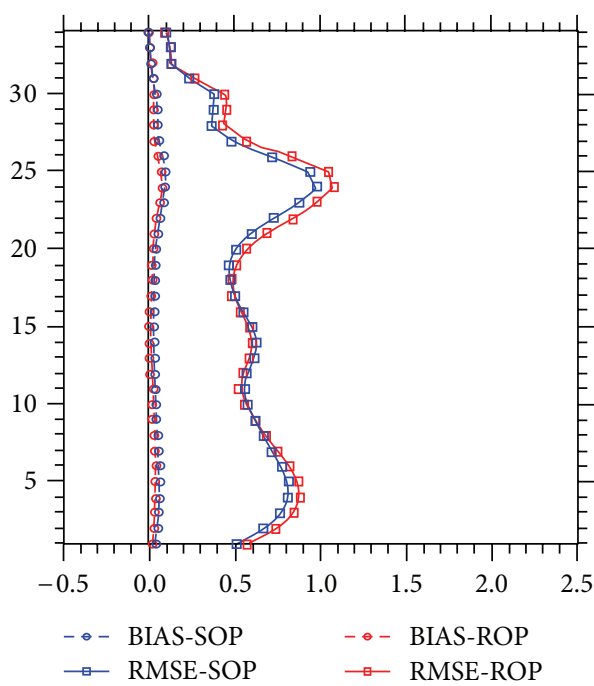

(g)

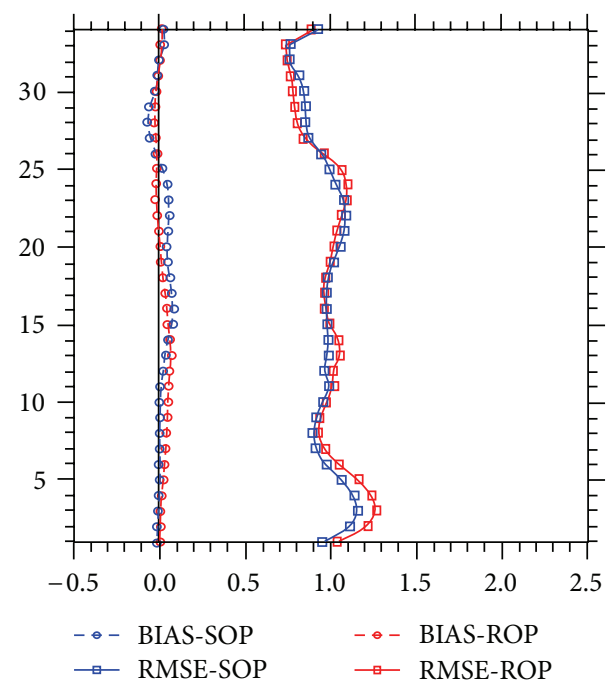

(h)

FIGURE 5: Bias and RMS error in temperature ((a), (b)), water vapor ((c), (d)), $u$-wind component $\left(\mathrm{m} \mathrm{s}^{-1}\right)((\mathrm{e}),(\mathrm{f}))$, and $v$-wind component $\left(\mathrm{m} \mathrm{s}^{-1}\right)((\mathrm{g}),(\mathrm{h}))$ of analyses (left column) and 12-h forecasts (right column) averaged over 1800 UTC 11 June and 1200 UTC 15 June 2002.

11 to 1200 UTC 15 June. The analysis at every $6 \mathrm{~h}$ is used to initialize a $24 \mathrm{~h}$ forecast. To better understand the impact of MTG-IRS retrieved profiles, three types of analysis differences are computed: (1) the analysis of assimilating simulated observation profiles (SOP) minus the first guess; (2) the results of assimilating simulated observations and MTG-IRS retrievals (SOP-RPtq or SOP-RPq) minus the results of SOP; (3) the analysis minus the "truth." The observation number of MTG-IRS retrieval profiles is much larger than that of the conventional observations in SOP. We expect that the MTGIRS observations will have a greater impact on analysis. The analysis should be more accurate when both simulated conventional observations and MTG-IRS retrievals are assimilated compared to the analysis produced by assimilating only simulated conventional observations, given that the MTGIRS retrievals are of high qualities.

The analysis at the beginning of the cycle at 1800 UTC 11 June is shown in detail since all experiments have the same background at that time. Figures 6 to 8 give the analysis differences in temperature $(T)$, water vapor mixing ratio $(q)$, and horizontal $u$-wind at $850 \mathrm{hPa}$ among the experiments SOP6hc, SOP-RPtq-6hc, control, and nature run, respectively. Assimilation of the MTG-IRS temperature and humidity retrievals (SOP-RPtq-6hc) leads to significant adjustments in $T, q$, and $u$ fields (Figures $6(\mathrm{~b}), 7(\mathrm{~b})$, and $8(\mathrm{~b})$ ).

In the background, there is a positive temperature bias along the Rocky Mountains (Figure 6(e)). Thus, negative analysis differences shown in Figures 6(a) and 6(b) indicate MTG-IRS has a larger impact than conventional observations. Comparing Figures 6(c) and 6(e), it is found that conventional observations help to improve the temperature analysis in Nebraska. Comparing Figures 6(c), 6(d), and 6(e), it is found that MTG-IRS observations help to improve the temperature analysis from Texas to Nebraska along the mountain range.
The MTG-IRS has a larger impact than conventional observations in humidity analysis compared Figure 7(b) (or Figure 7(c) to Figure 7(a)). The humidity increment in SOPRPtq-6hc and SOP-RPq-6hc is very similar (Figures 7 (b) and $7(\mathrm{c})$ ). Comparing Figures 7 (a) to $7(\mathrm{f})$, it is seen that the humidity analysis in SOP-6hc is improved near the Rocky Mountains from Texas to Nebraska. In SOP-RPtq-6hc, the humidity analysis along the Rocky Mountains is much improved when moisture retrievals are assimilated, but slightly degraded in south Texas region due to the dry bias in the retrievals.

MTG-IRS retrievals have little impact on wind analysis comparing with SOP-6hc. In SOP-6hc, the $u$ analysis is improved in the regions between Texas and Oklahoma. The maximum wind increment is about $3 \mathrm{~m} \mathrm{~s}^{-1}$ (Figure 8(a)). In SOP-RPtq-6hc, the maximum wind increment is about $1 \mathrm{~m} \mathrm{~s}^{-1}$ (Figure 8(b)). The wind increments in SOP-RPtq6hc are derived from the statistical balance relation between temperature and wind in the background error covariance. The $u$ analysis in the central Texas region is significantly improved when comparing Figures $8(\mathrm{c})-8(\mathrm{~d})$ to $8(\mathrm{e})$. The $v$ analysis is improved in southeast Texas (figure not shown).

The analyses at other times have also been checked. Analyses show that assimilation of simulated conventional observations improves analyses of $T, q, u$, and $v$ compared with the control experiment. Compared with SOP-6hc, the assimilation of MTG-IRS temperature and humidity retrievals significantly improves temperature and water vapor analysis, and slightly improves the wind analysis.

Using the nature run as the "truth" the RMS errors of the analysis and the forecast are computed. In each 6-hourly cycling experiment, there are 16 analyses and 16 forecasts during the cycling period. The averaged RMS errors at analysis time are shown in Figure 9. As expected, assimilating the simulated observations as well as the MTG-IRS retrievals 


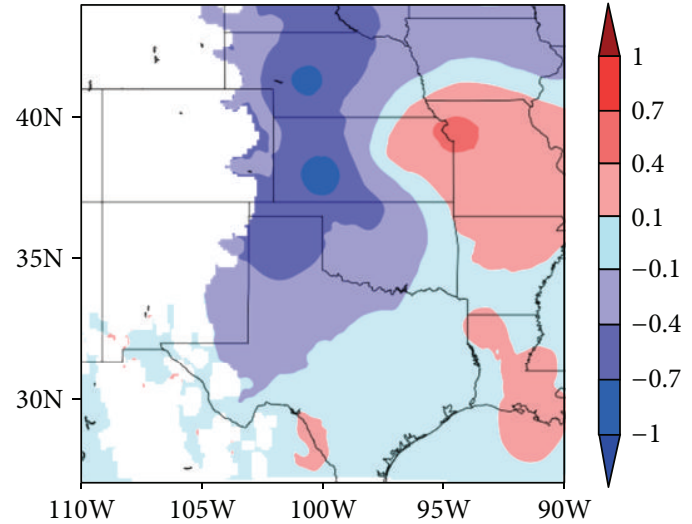

(a)

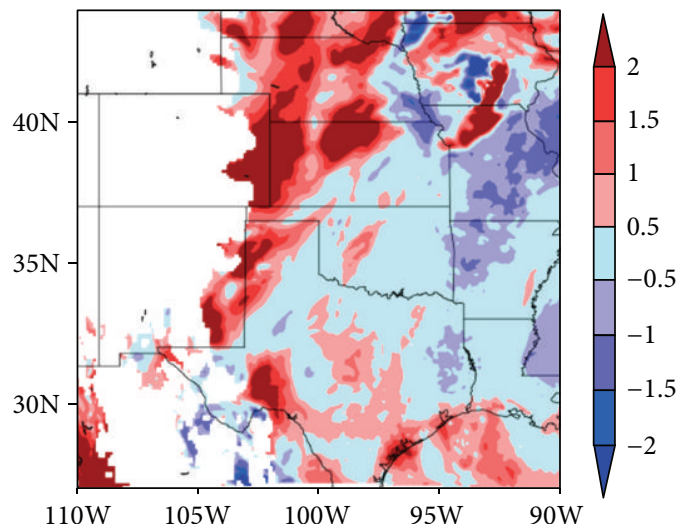

(c)

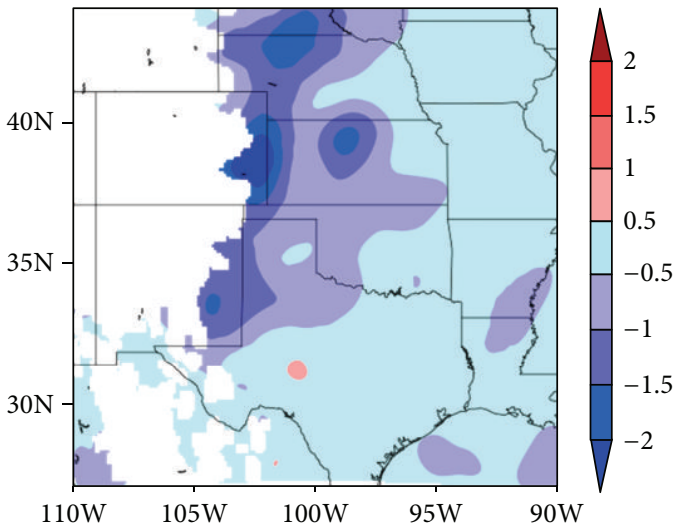

(b)

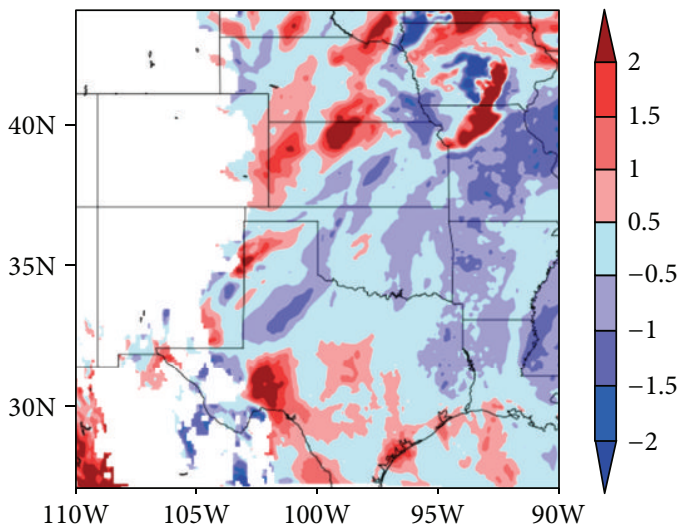

(d)

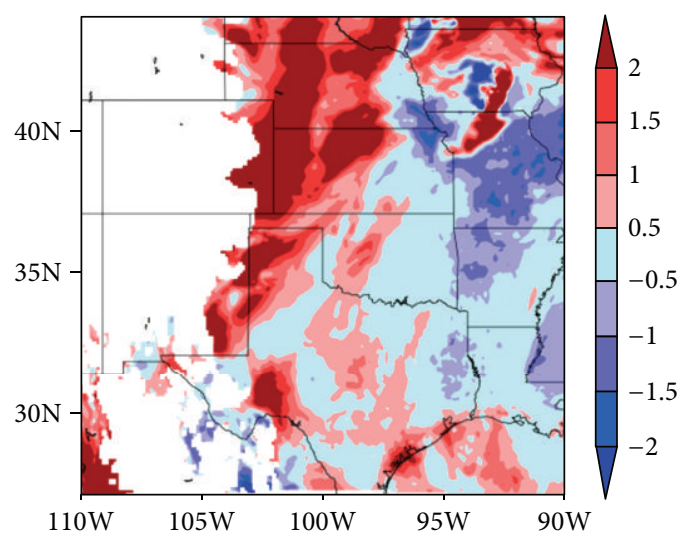

(e)

FIGURE 6: Differences of the $850 \mathrm{hPa}$ temperature T (unit: K) between (a) SOP-6hc and control, (b) SOP-RPtq-6hc and SOP-6hc, (c) SOP-6hc and "truth", (d) SOP-RPtq-6hc and "truth", and (e) control and "truth", valid at 1800 UTC 11 June.

(SOP-RPtq-6hc) significantly reduces the analysis errors compared to the control experiment. Comparing SOP-RPtq$6 \mathrm{hc}$ with SOP-RPq-6hc, assimilating both temperature and moisture retrievals yields the better temperature analyses and slightly improves the $u$ and $v$ analyses; similar improvements in moisture analysis are obtained.

The above results indicate the assimilation of MTGIRS temperature and humidity profiles significantly reduces the temperature and the water vapor analysis error in the low-level atmosphere. Past research shows that the realistic mesoscale details of the horizontal variations in low-level moisture and temperature in analyses would help to improve forecast skills for convective events $[8,12,13]$. Thus, the forecast accuracy is expected to be increased due to the improvements of the analyses.

The averaged RMS errors of the $12 \mathrm{~h}$ forecast are shown in Figure 10. It is seen that the assimilation of simulated conventional observations improves the temperature, humidity, 


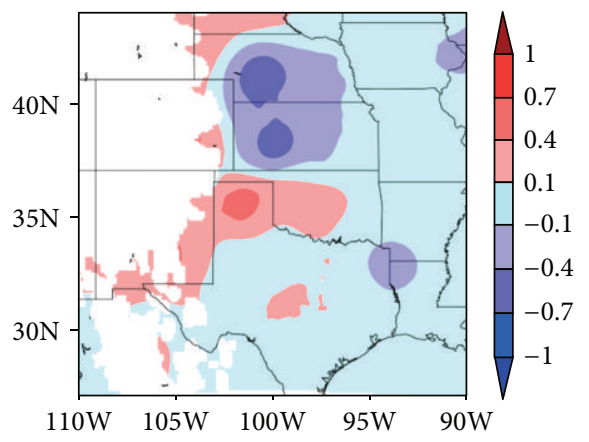

(a)

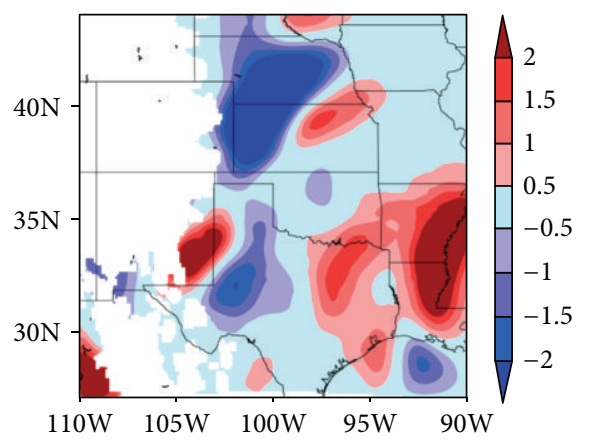

(c)

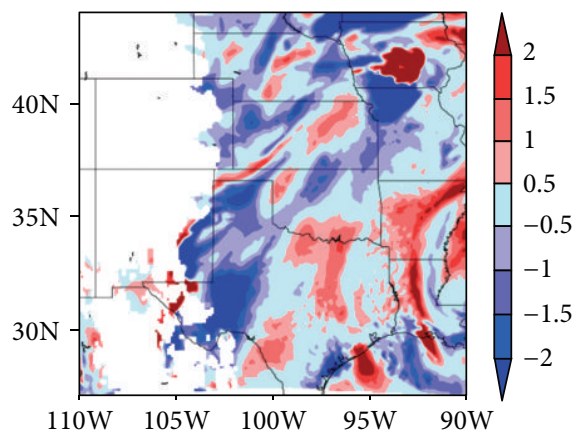

(e)

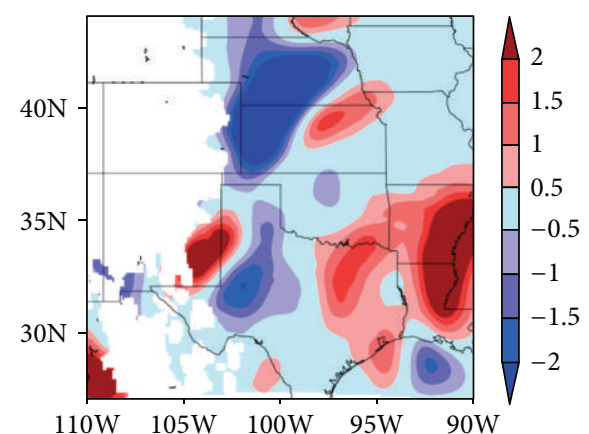

(b)

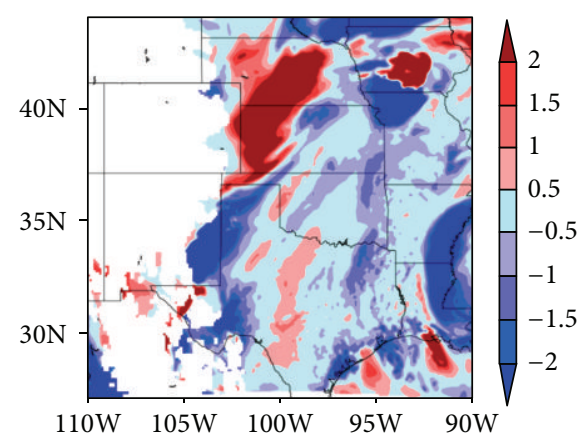

(d)

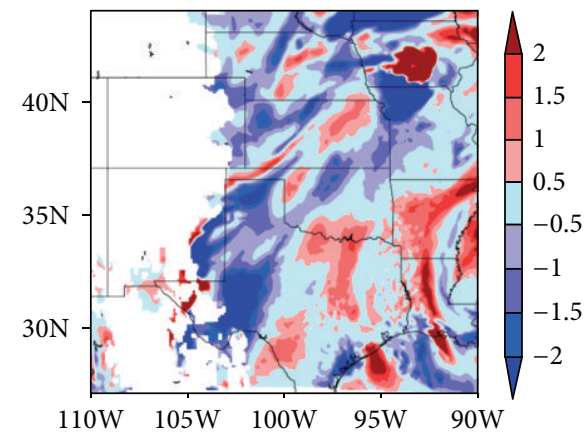

(f)

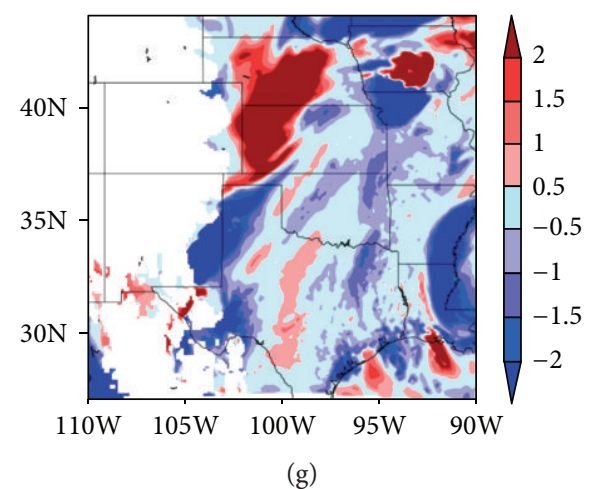

FIGURE 7: Differences of the $850 \mathrm{hPa}$ water vapor mixing ratio $q$ (unit: $\mathrm{g} \mathrm{kg}^{-1}$ ) between (a) SOP-6hc and control, (b) SOP-RPq-6hc and SOP6 he and (c) SOP-RPtq-6hc and SOP-6hc, (d) SOP-6hc and "truth," (e) SOP-RPq-6hc and "truth", (f) SOP-RPtq-6hc and "truth," and (g) Control and "truth", valid at 1800 UTC 11 June. 


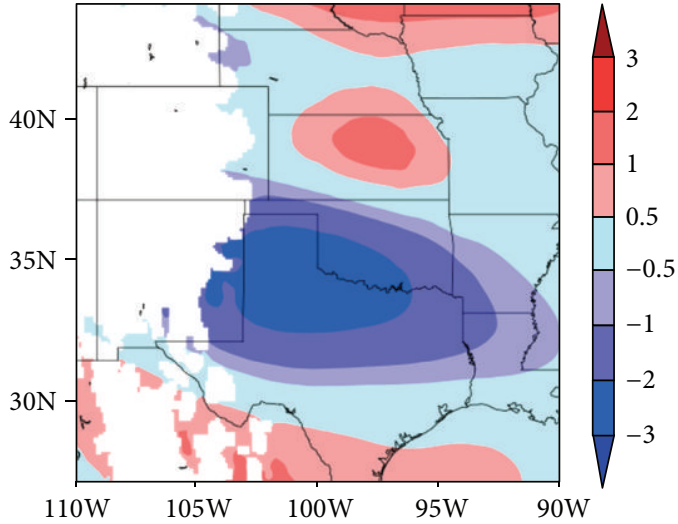

(a)

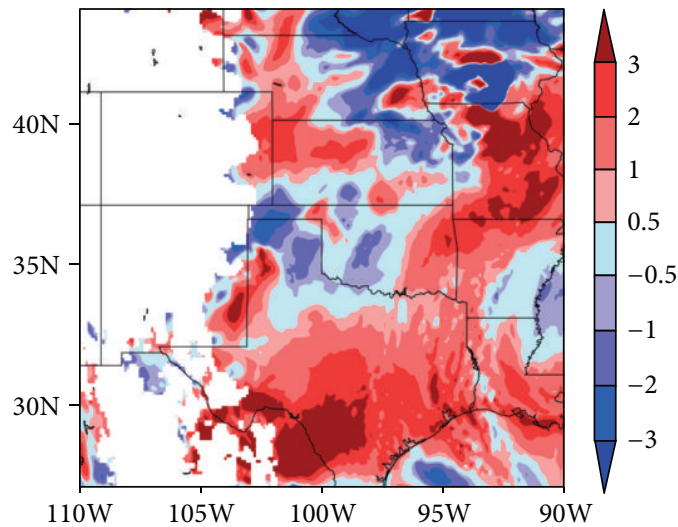

(c)

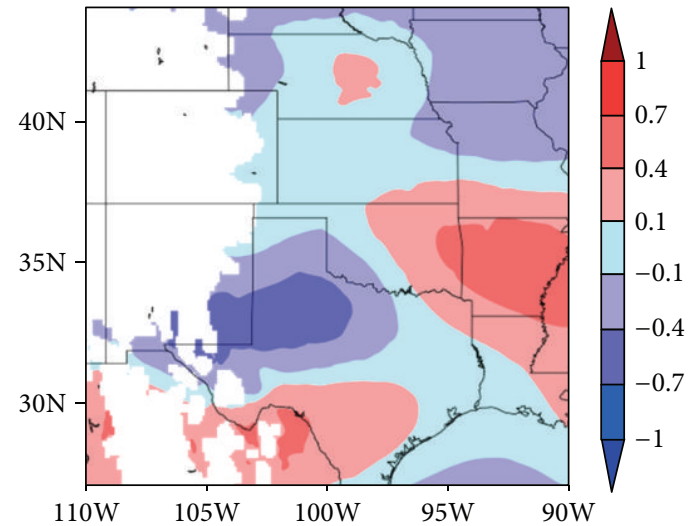

(b)

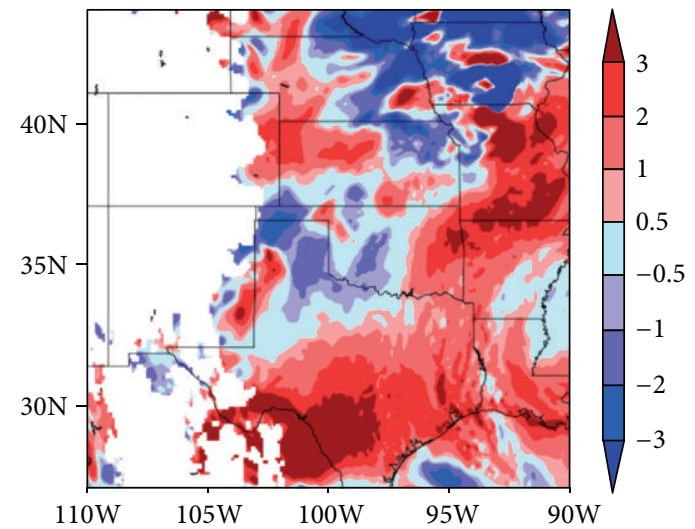

(d)

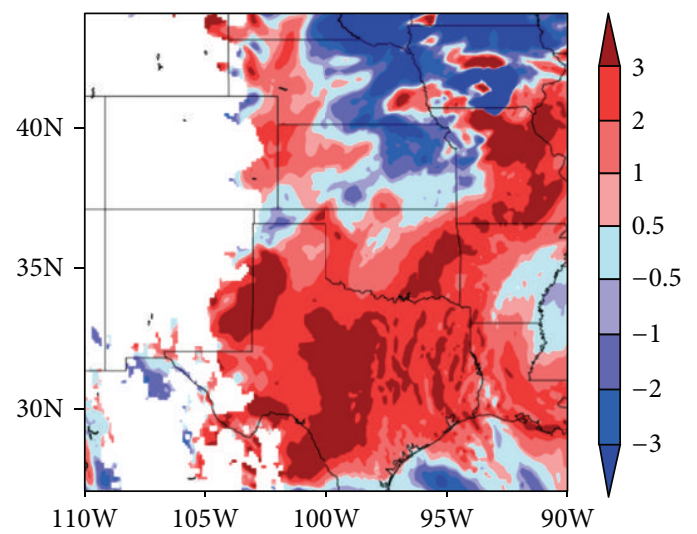

(e)

Figure 8: Differences of the $850 \mathrm{hPa} u$ wind (unit: $\mathrm{m} \mathrm{s}^{-1}$ ) between (a) SOP-6hc and control, (b) SOP-RPtq-6hc and SOP-6hc, (c) SOP-6hc and "truth," (d) SOP-RPtq-6hc and "truth," and (e) control and "truth," valid at 1800 UTC 11 June.

and wind forecasts over the control run. The MTG-IRS humidity observations (SOP-RPq-6hc) improve the moisture and upper level wind forecast, but they slightly degrade the temperature forecast in the lower-level atmosphere. Compared to simulated conventional observations (SOP-6hc), when the MTG-IRS temperature and humidity observations (SOPRPtq-6hc) are assimilated, the temperature and moisture forecasts are improved significantly over that of wind.
The maximum potential impact of perfect observations of MTG-IRS on analyses can be found in POP in which the true soundings of temperature, humidity, and $u / v$ wind at the same times and locations of the MTG-IRS retrievals are assimilated. Using the accurate observations produces the best analyses and forecasts of $u, v, T$, and $q$ among all the cycling experiments (Figures 9 and 10). This suggests that if MTG-IRS can provide accurate retrievals of $T, q$, as well as 


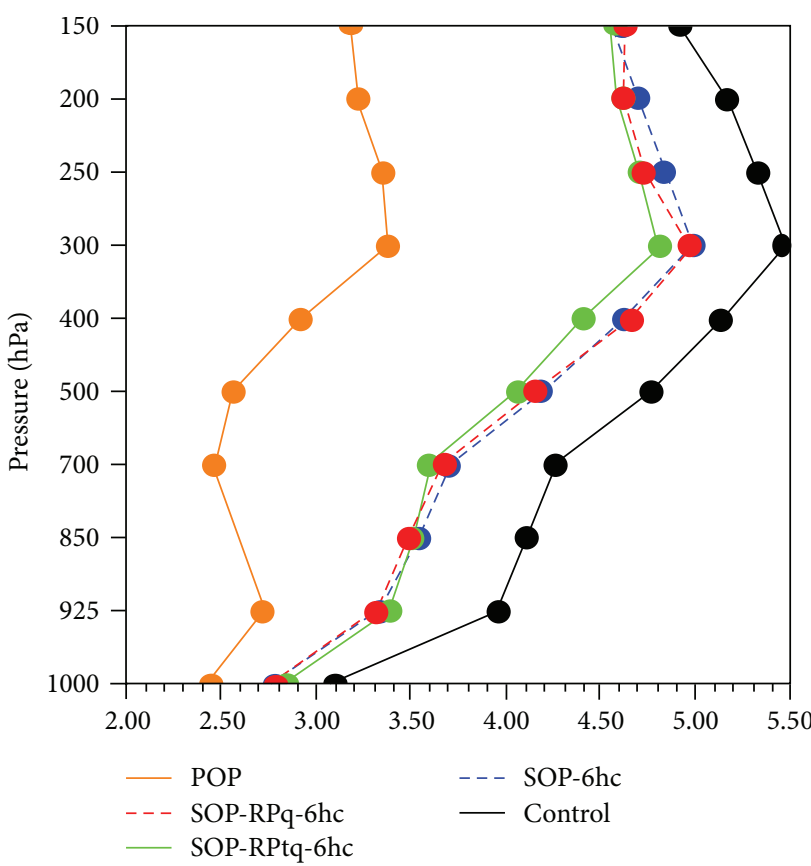

(a)

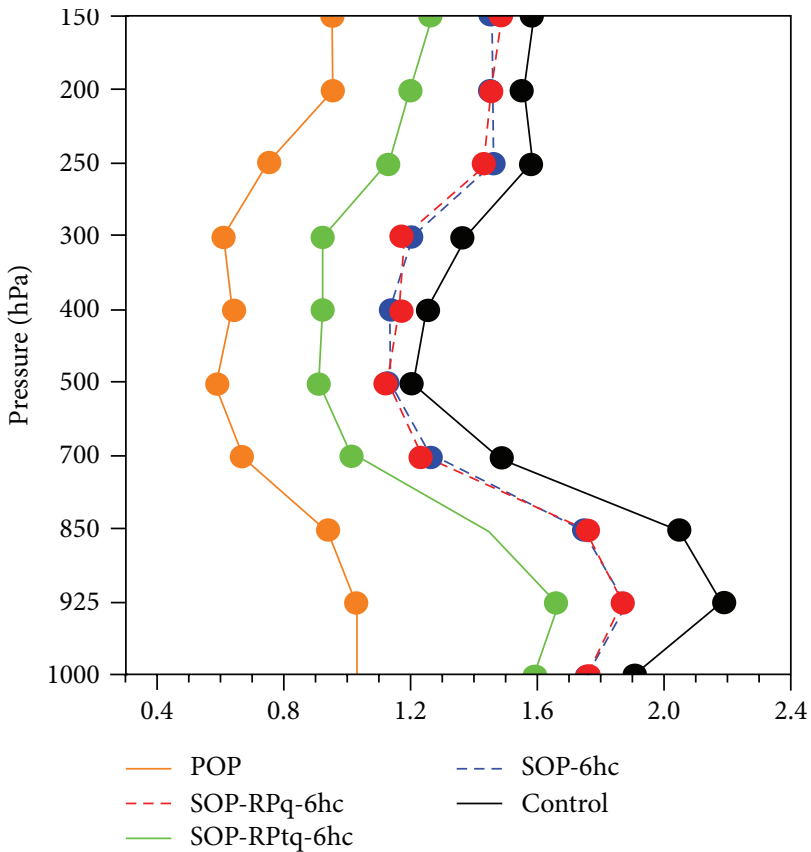

(c)

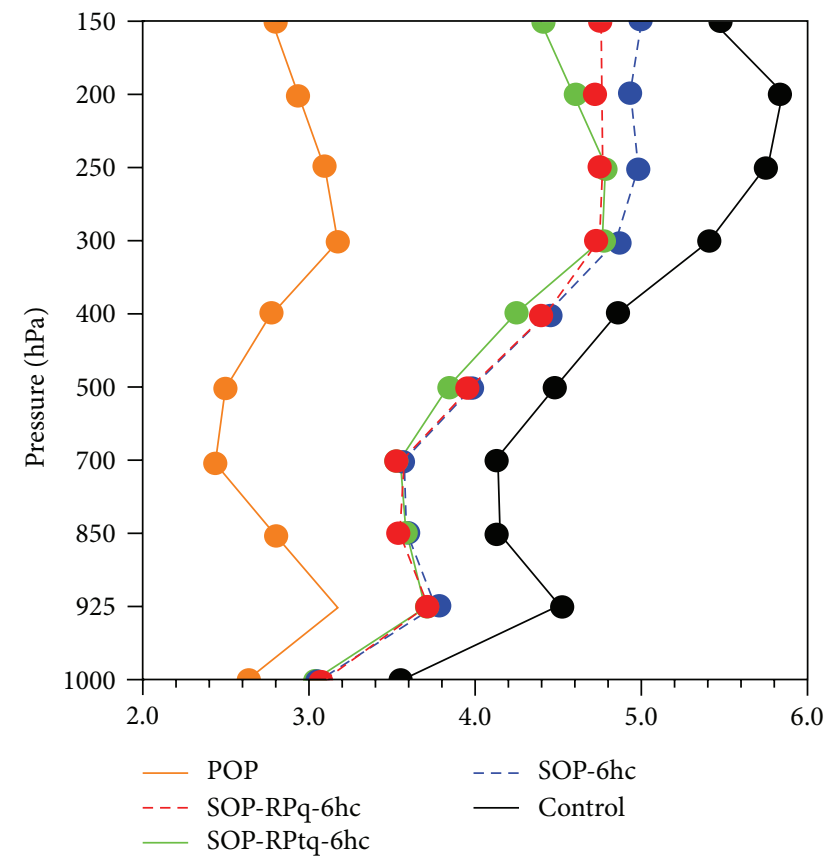

(b)

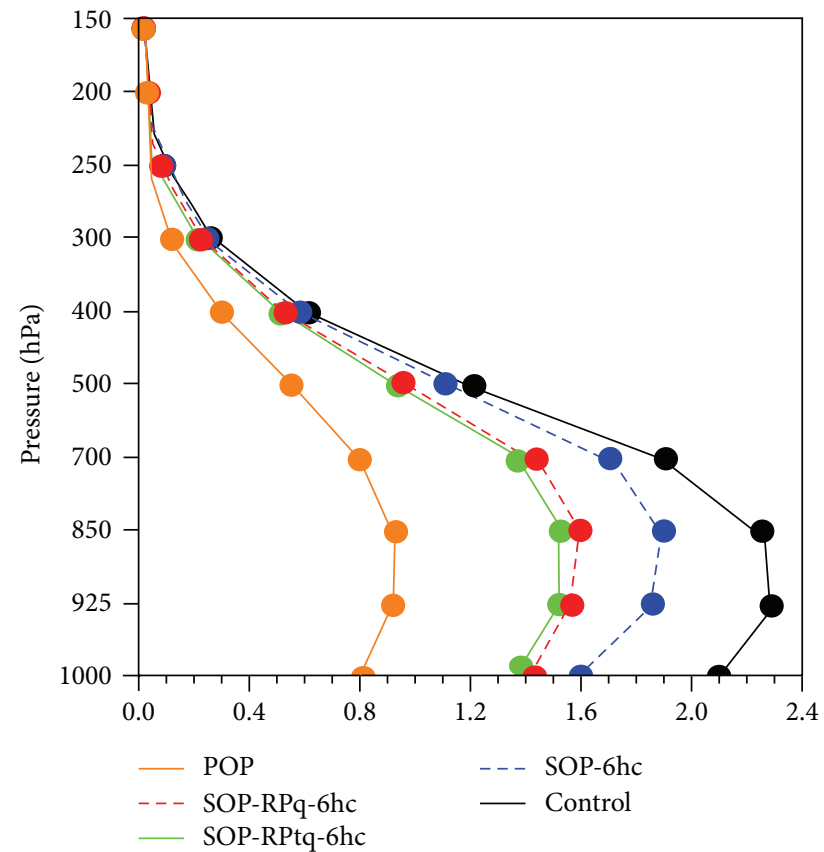

(d)

FIgURE 9: Averaged RMS errors for experiments POP, SOP-RPq-6hc, SOP-RPtq-6hc, SOP-6hc, and control at analysis time. (a) Zonal wind component $u\left(\mathrm{~m} \mathrm{~s}^{-1}\right)$, (b) meridional wind component $v\left(\mathrm{~m} \mathrm{~s}^{-1}\right)$, (c) temperature $T(\mathrm{~K})$, and (d) water vapor mixing ratio $q\left(\mathrm{~g} \mathrm{~kg}^{-1}\right)$.

winds in all regions, it will impose great values in the analysis and forecast.

4.5. Hourly Cycling Experiments. Since high temporal resolution MTG-IRS retrievals can be obtained, it is useful to test their impact on the regional forecasts. Two 1-hourly cycling experiments have been carried out. The RMS errors of the experiments (figures not shown) indicate that increasing assimilation frequency yields only slight improvements on the analysis compared to 6-hourly cycling experiments. The $12 \mathrm{~h}$ forecast accuracy of temperature and moisture are also improved, while a neutral impact on the wind forecast is observed. However, it is found that the hourly cycling has a noticeable positive impact on precipitation forecasts, as will be shown in Section 4.6. 


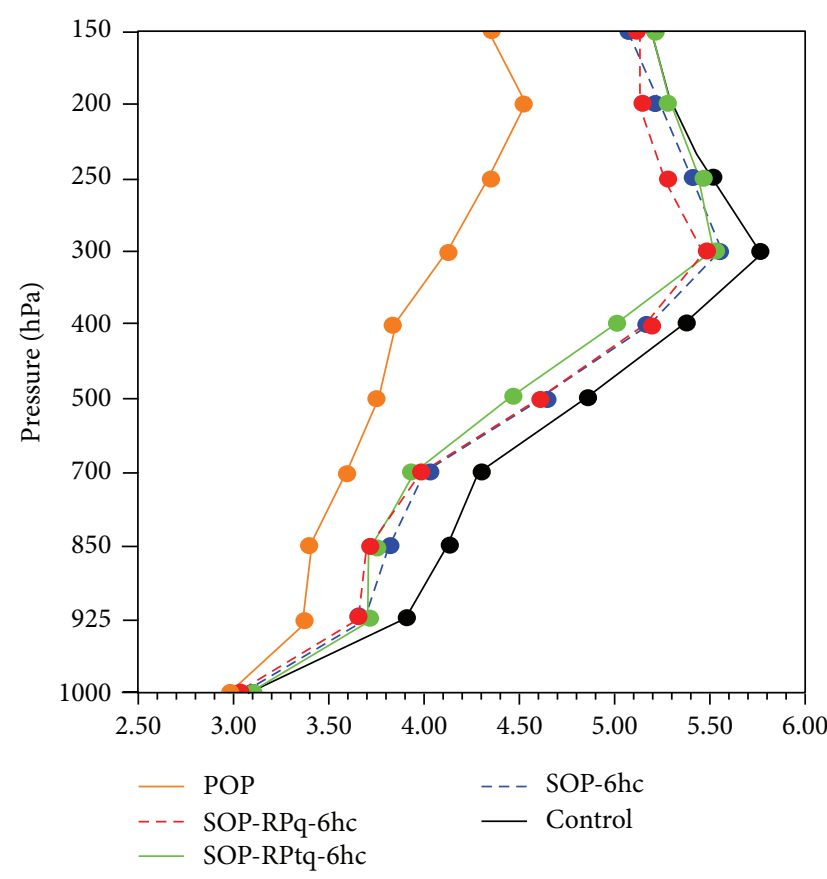

(a)

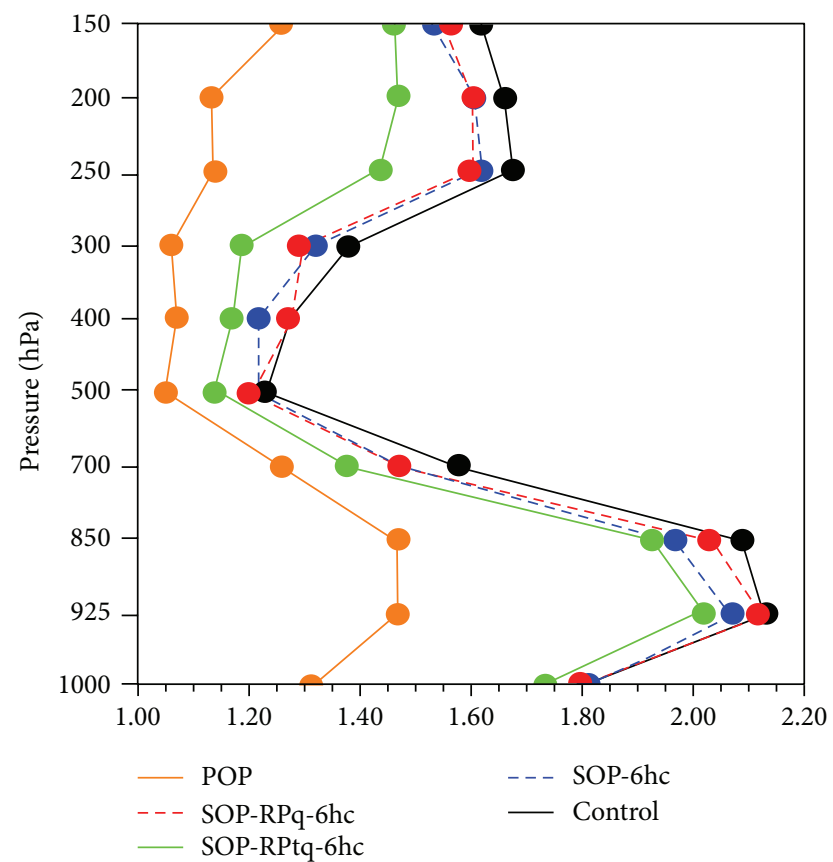

(c)

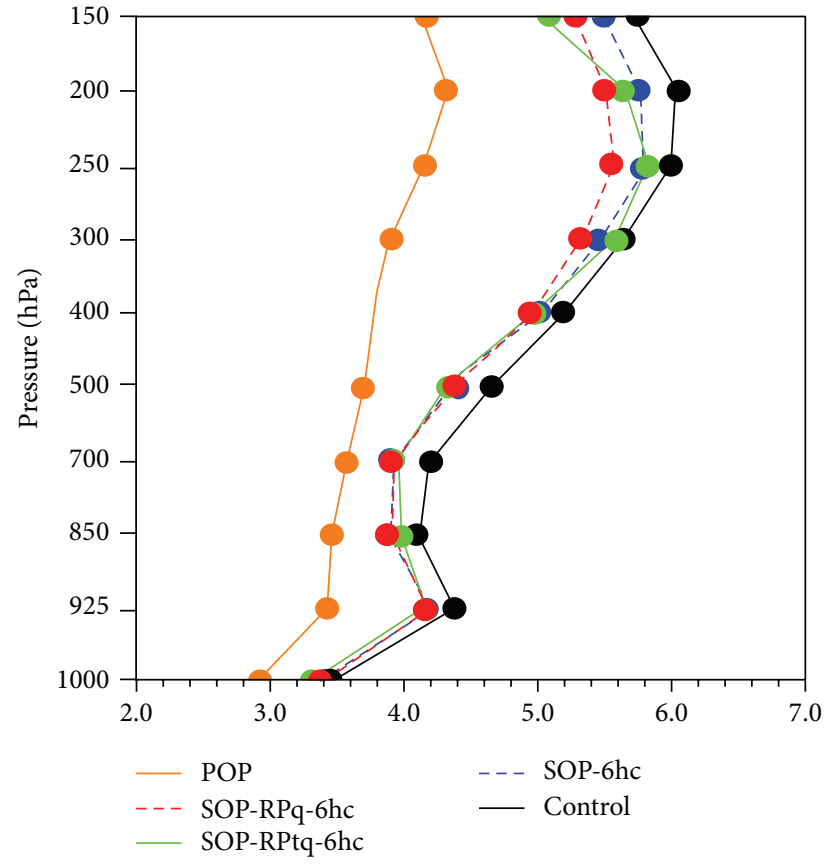

(b)

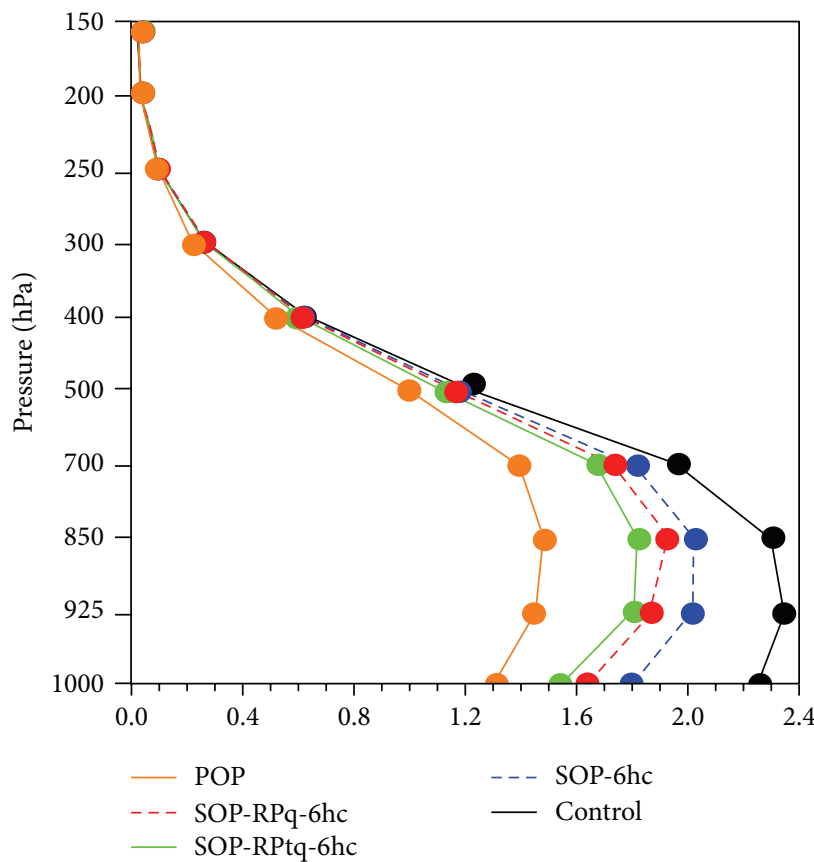

(d)

Figure 10: Same as Figure 9 but for $12 \mathrm{~h}$ forecast.

4.6. Precipitation Forecast Verification. For the first case, the equitable threat scores (ETS) and frequency bias for $18 \mathrm{~h}$ accumulated rainfall with thresholds of $10 \mathrm{~mm}, 30 \mathrm{~mm}, 50 \mathrm{~mm}$, $65 \mathrm{~mm}, 80 \mathrm{~mm}$, and $100 \mathrm{~mm}$ are shown in Figure 11. The forecasts are from the analysis at the beginning of the cycle at 1800 UTC 11 June, so that there is no difference between the forecast from 1- and 6-hourly cycling experiment. Figure 11 shows that SOP-6hc improves only the $10 \mathrm{~mm}$ precipitation forecasts. It is evident that assimilating MTG-IRS temperature and humidity retrievals (SOP-RPtq-6hc) substantially improves the precipitation forecast skill. The MTG-IRS humidity retrievals improve the rainfall forecast skill below the threshold of $30 \mathrm{~mm}$. The frequency bias shows the rainfall frequency forecast in experiments SOP-RPtq-6hc, and SOP$\mathrm{RPq}-6 \mathrm{hc}$ is comparable to the control experiment, but adding MTG-IRS temperature and humidity yields better rainfall 


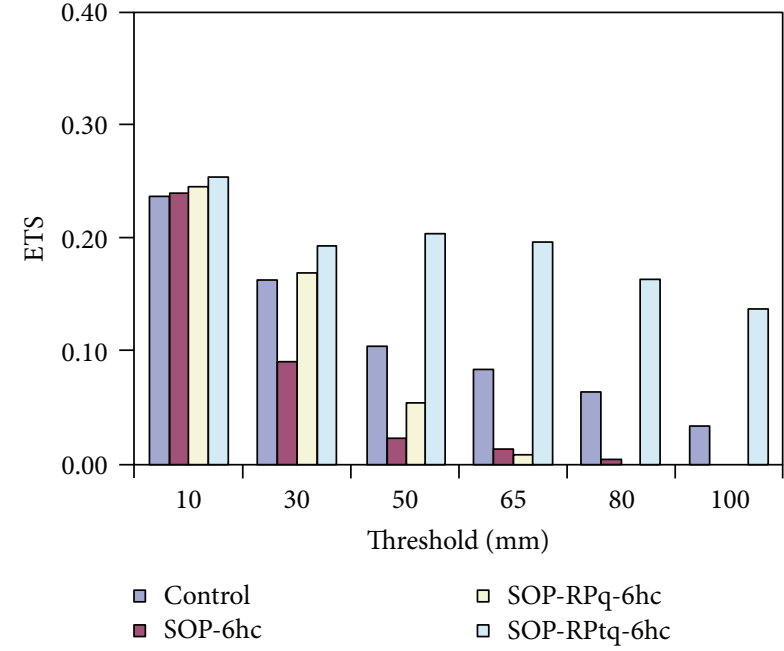

(a)

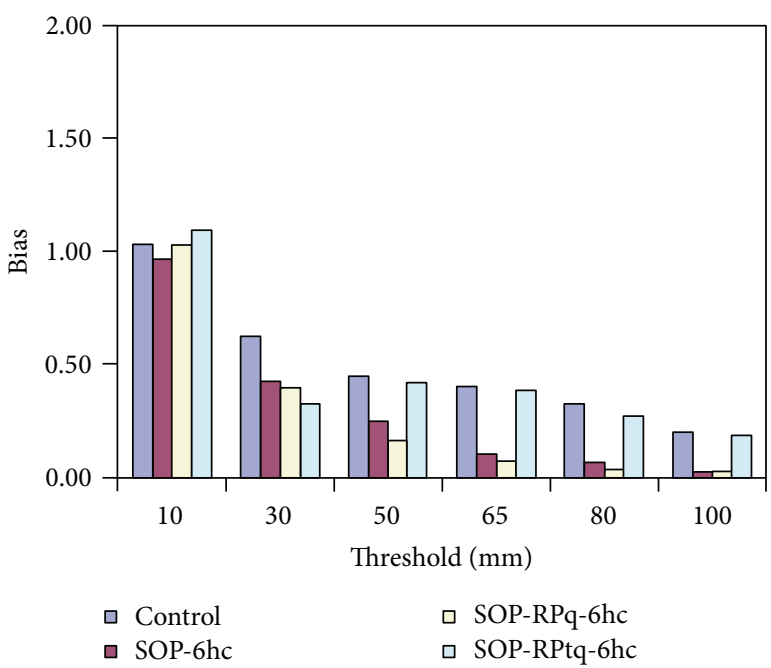

(b)

FIGURE 11: (a) ETS and (b) frequency bias of $18 \mathrm{~h}$ accumulated precipitation for experiments control, SOP-6hc, SOP-RPq-6hc, and SOP-RPtq$6 \mathrm{hc}$, valid at 1200 UTC 12 June.

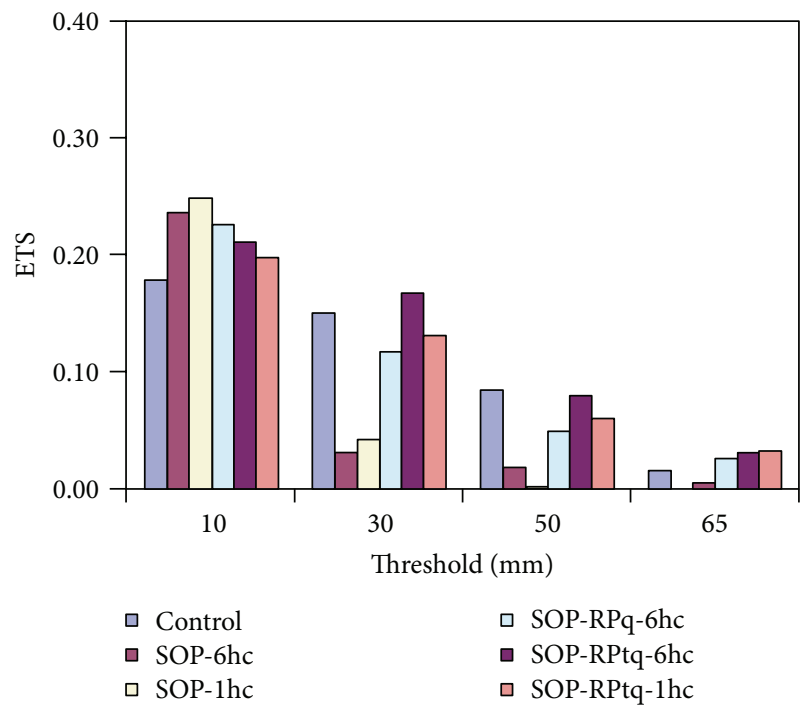

(a)

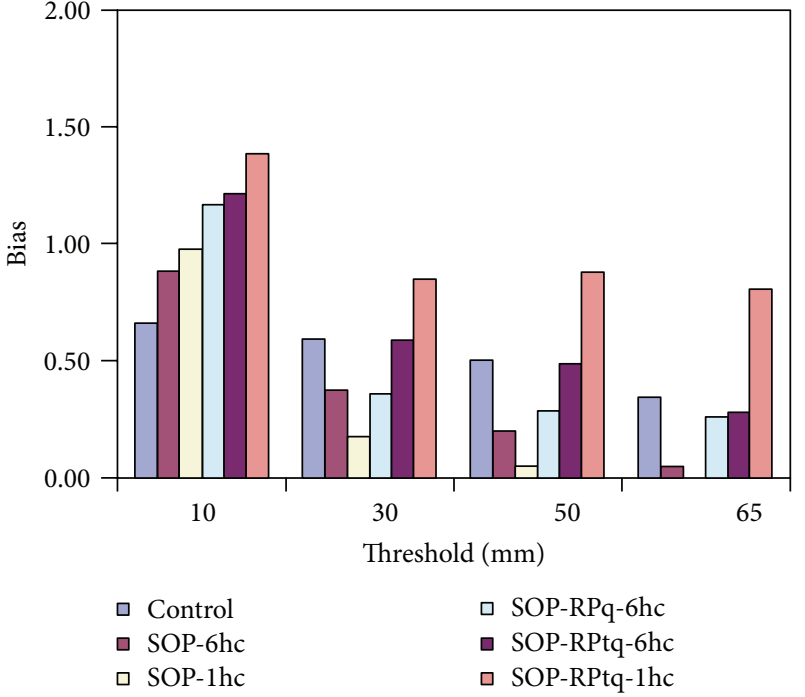

(b)

FIGURE 12: (a) ETS and (b) frequency bias of $18 \mathrm{~h}$ accumulated precipitation for experiments control, SOP-6hc, SOP-1hc, SOP-RPq-6hc, SOPRPtq-6hc, and SOP-RPtq-1hc, valid at 1200 UTC 13 June, 2002.

frequency forecast than assimilating simulated conventional observations alone (SOP-6hc).

For the second convective event, ETS and frequency bias for $18 \mathrm{~h}$ accumulated rainfall with thresholds of $10 \mathrm{~mm}$, $30 \mathrm{~mm}, 50 \mathrm{~mm}$, and $65 \mathrm{~mm}$ are shown in Figure 12. In general, MTG-IRS temperature and moisture retrievals (SOP-RPtq6hc) have positive impacts on precipitation forecasts at thresholds $10 \mathrm{~mm}$ and $30 \mathrm{~mm}$. The rainfall forecast frequency for experiments SOP-RPtq-6hc and SOP-RPtq-1hc is better than other data assimilation experiments when threshold is larger than $10 \mathrm{~mm}$.

For the third convective event, the threat scores (figures not shown) for $18 \mathrm{~h}$ accumulated rainfall show that MTG-IRS temperature and moisture retrievals (SOP-RPtq-6hc) have positive impacts on precipitation forecast at threshold $10 \mathrm{~mm}$. The rainfall forecast frequency for experiments SOP-RPtq$6 \mathrm{hc}$ and SOP-RPtq-1hc is better than experiments assimilating only the simulated conventional data.

The averaged ETS and frequency bias of all 16 forecasts of $18 \mathrm{~h}$ accumulated rainfall with thresholds of $10 \mathrm{~mm}, 30 \mathrm{~mm}$, $50 \mathrm{~mm}, 65 \mathrm{~mm}, 80 \mathrm{~mm}$, and $100 \mathrm{~mm}$ during the entire cycling period are shown in Figure 13. On average, MTG-IRS temperature and humidity retrievals (SOP-RPtq-6hc and SOPRPtq-1hc) significantly improve precipitation forecast skill at thresholds larger than $10 \mathrm{~mm}$. All the experiments improve ETS and bias at threshold $10 \mathrm{~mm}$. In general, assimilating 


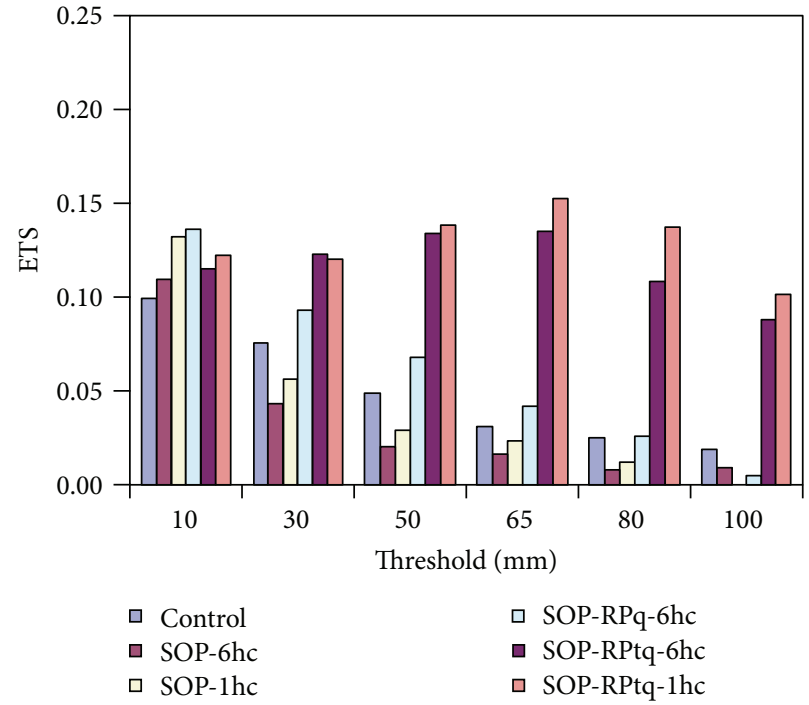

(a)

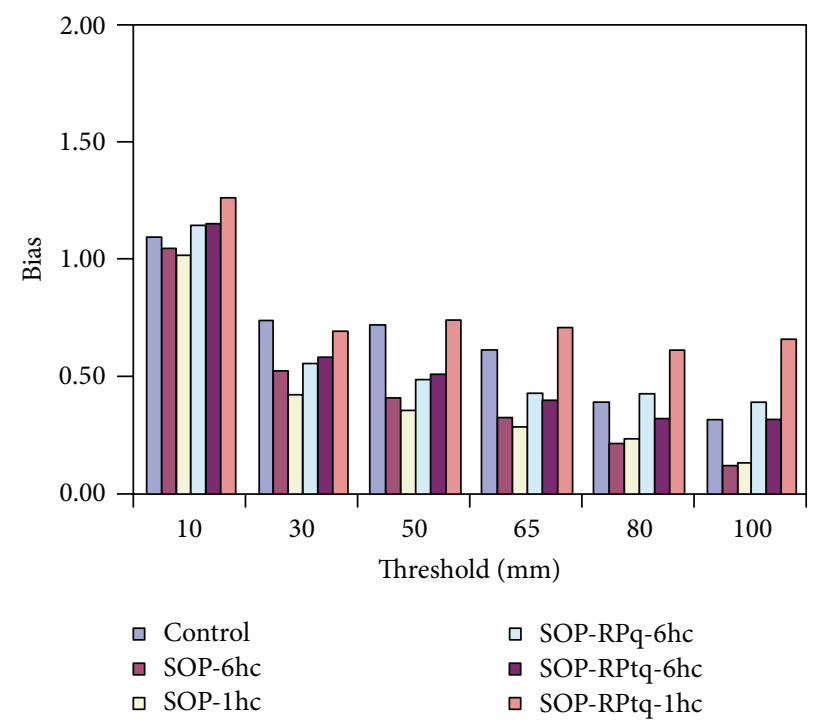

(b)

FIGURE 13: (a) Averaged ETS and (b) averaged frequency bias of 16 forecasts of $18 \mathrm{~h}$ accumulated precipitation for experiments control, SOP6hc, SOP-1hc, SOP-RPq-6hc, SOP-RPtq-6hc, and SOP-RPtq-1hc.

hourly MTG-IRS observations yields better precipitation forecasts than using 6-hourly data in terms of ETS and frequency bias.

\section{Summary and Discussion}

An OSSE system was designed and conducted to document the added value of temperature and water vapor observations derived from the MTG-IRS to regional forecasts, especially to precipitation prediction. The OSSE system was characterized by the nature run using the MM 5 model with $4 \mathrm{~km}$ resolution, and the $12 \mathrm{~km}$ resolution 6/1-hourly cycling data assimilation and forecast experiments using the WRF model, and a pair of calibration experiments. The nature run and data assimilation and forecast experiments employed two different mesoscale models to reduce the identical twin problem. The calibration run was conducted to show the performance of the OSSE system. The OSSE calibration showed that assimilation of real or simulated conventional observations gives similar error statistics in analyses and forecasts, indicating that the developed OSSE system performed reasonably well.

Three convective cases over US Great Plains were selected to show MTG-IRS's impact on high-impact weather forecasts. The results showed that the MTG-IRS retrievals have positive impacts on the analyses and the subsequent forecasts. The forecast skills for $T, q, u$, and $v$ are improved up to 12 hours. The $18 \mathrm{~h}$ quantitative precipitation forecasts are also improved, in particular for heavy precipitation events. Further positive impacts are obtained by assimilating hourly MTG-IRS temperature and moisture retrievals.

Although the preliminary results from case studies are promising, we note that the current OSSE configuration may overestimate the impact of MTG-IRS retrievals. For conventional observations, the same observation statistics are used in generating simulated observations and data assimilation procedure. For MTG-IRS retrievals, the "true" observation error (against nature run) is used. Currently, only the conventional data are assimilated in control experiments. These can lead to an overoptimistic assessment of the observation impact as well. Other issues, for example, sensitivity to forecast model resolution, need to be further studied as well. In future work, more cases, radar observations, and other types of satellite data (e.g., microwave radiance and infrared images) will be simulated in the OSSE system to give a more realistic estimation of MTG-IRS data on regional forecasts.

\section{Conflict of Interests}

The authors of the paper do not have a direct financial relation with the commercial identities mentioned in the paper.

\section{Acknowledgments}

The authors would like to thank Stephen A. Tjemkes and Rolf Stuhlmann (EUMETSAT) for providing MTG-IRS retrievals and their valuable suggestions and comments on this work. NCAR is sponsored by the National Science Foundation. Any opinions, findings, and conclusions or recommendations expressed in this publication are those of the authors and do not necessarily reflect the views of the National Science Foundation.

\section{References}

[1] T. H. Zapotocny, J. A. Jung, J. F. Le Marshall, and R. E. Treadon, "A two-season impact study of satellite and in situ data in the NCEP global data assimilation system," Weather and Forecasting, vol. 22, no. 4, pp. 887-909, 2007. 
[2] D. P. Dee, S. M. Uppala, A. J. Simmons, P. Berrisford, and P. Poli, "The ERA-Interim reanalysis: configuration and performance of the data assimilation system," Quarterly Journal of the Royal Meteorological Society, vol. 137, pp. 553-597, 2011.

[3] P. Bauer, A. J. Geer, P. Lopez, and D. Salmond, "Direct 4DVar assimilation of all-sky radiances. Part I: implementation," Quarterly Journal of the Royal Meteorological Society, vol. 136, no. 652, pp. 1868-1885, 2010.

[4] F. Weng, T. Zhu, and B. Yan, "Satellite data assimilation in numerical weather prediction models. Part II: uses of rainaffected radiances from microwave observations for hurricane vortex analysis," Journal of the Atmospheric Sciences, vol. 64, no. 11, pp. 3910-3925, 2007.

[5] T. Vukicevic, T. Greenwald, M. Zupanski, D. Zupanski, T. Vonder Haar, and A. S. Jones, "Mesoscale cloud state estimation from visible and infrared satellite radiances," Monthly Weather Review, vol. 132, no. 12, pp. 3066-3077, 2004.

[6] T. Vukicevic, M. Sengupta, A. S. Jones, and T. Vonder Haar, "Cloud-resolving satellite data assimilation: information content of IR window observations and uncertainties in estimation," Journal of the Atmospheric Sciences, vol. 63, no. 3, pp. 901919, 2006.

[7] S. Tjemkes, J. Grandell, R. Borde, and R. Stuhlmann, "Capabilities of the MTG-IRS candidate candidate mission to depict horizontal moisture structures in Fourier transform spectroscopy/hyperspectral imaging and sounding of the environment," OSA Technical Digest Series (CD) (Optical Society of America, 2007), paper JWA13, 2007.

[8] N. A. Crook, "Sensitivity of moist convection forced by boundary layer processes to low-level thermodynamic fields," Monthly Weather Review, vol. 124, no. 8, pp. 1767-1785, 1996.

[9] M. Xue and W. J. Martin, "A high-resolution modeling study of the 24 May 2002 dryline case during IHOP. Part II: horizontal convective rolls and convective initiation," Monthly Weather Review, vol. 134, no. 1, pp. 172-191, 2006.

[10] S. E. Koch, A. Aksakal, and J. T. McQueen, “The influence of mesoscale humidity and evapotranspiration fields on a model forecast of a cold-frontal squall line," Monthly Weather Review, vol. 125, pp. 384-409, 1997.

[11] D. B. Parsons, M. A. Shapiro, and E. Miller, "The mesoscale structure of a nocturnal dryline and of a frontal-dryline merger," Monthly Weather Review, vol. 128, no. 11, pp. 3824-3838, 2000.

[12] T. M. Weckwerth, "The effect of small-scale moisture variability on thunderstorm initiation," Monthly Weather Review, vol. 128, no. 12, pp. 4017-4030, 2000.

[13] T. M. Weckwerth, D. B. Parsons, S. E. Koch et al., "An overview of the International $\mathrm{H}_{2} \mathrm{O}$ project (IHOP_2002) and some preliminary highlights," Bulletin of the American Meteorological Society, vol. 85, no. 2, pp. 253-277.

[14] M. Masutani, J. S. Woollen, S. J. Lord et al., "Observing system simulation experiments at the National Centers for Environmental Prediction," Journal of Geophysical Research, vol. 115, no. D7, Article ID D07101, 2010.

[15] L. Zhang and Z. Pu, "An Observing System Simulation Experiment (OSSE) to assess the impact of Doppler wind lidar (DWL) measurements on the numerical simulation of a tropical cyclone," Advances in Meteorology, vol. 2010, Article ID 743863, 14 pages, 2010.

[16] S.-H. Chen, J.-Y. Chen, W.-Y. Chang, P.-L. Lin, P.-H. Lin, and W.-Y. Sun, "Observing system simulation experiment: development of the system and preliminary results," Journal of Geophysical Research, vol. 116, Article ID D13202, 2011.

[17] X.-Y. Huang, F. Gao, N. Jacobs, and H. Wang, "Assimilation of wind speed and direction observations: a new formulation and results from idealized experiments," Tellus A, vol. 65, Article ID 19936, 2013.

[18] D. T. Dawson and M. Xue, "Numerical forecasts of the 15-16 June 2002 southern plains mesoscale convective system: impact of mesoscale data and cloud analysis," Monthly Weather Review, vol. 134, no. 6, pp. 1607-1629, 2006.

[19] H. Wang, J. Sun, X. Zhang, X.-Y. Huang, and T. Auligné, "Radar data assimilation with WRF 4D-Var: part I. System development and preliminary testing," Monthly Weather Review, 2013.

[20] J. Dudhia, "A nonhydrostatic version of the Penn State-NCAR mesoscale model: validation tests and simulation of an Atlantic cyclone and cold front," Monthly Weather Review, vol. 121, no. 5, pp. 1493-1513, 1993.

[21] W. C. Skamarock, J. B. Klemp, J. Dudhia et al., "A description of the advanced research WRF version 3," NCAR Tech. Note TN475+STR, 2008.

[22] D. M. Barker, W. Huang, Y.-R. Guo, A. J. Bourgeois, and Q. N. Xiao, "A three-dimensional variational data assimilation system for MM5: implementation and initial results," Monthly Weather Review, vol. 132, pp. 897-914, 2004.

[23] X. Y. Huang, Q. Xiao, D. M. Barker et al., "Four-dimensional variational data assimilation for WRF: formulation and preliminary results," Monthly Weather Review, vol. 137, no. 1, pp. 299314, 2009.

[24] S. Y. Hong and H. L. Pan, "Nonlocal boundary layer vertical diffusion in a medium-range forecast model," Monthly Weather Review, vol. 124, no. 10, pp. 2322-2339, 1996.

[25] J. Reisner, R. M. Rasmussen, and R. T. Bruintjes, "Explicit forecasting of supercooled liquid water in winter storms using the MM5 mesoscale model," Quarterly Journal of the Royal Meteorological Society, vol. 124, no. 548, pp. 1071-1107, 1998.

[26] S. Hannon, L. L. Strow, and W. W. McMillan, "Atmospheric infrared fast transmittance models: a comparison of two approaches," in Optical Spectroscopic Techniques and Instrumentation for Atmospheric and Space Research II, vol. 2830 of Proceedings of SPIE, pp. 94-105, 1996.

[27] T. J. Schmit, M. M. Gunshor, W. P. Menzel, J. J. Gurka, J. $\mathrm{Li}$, and $\mathrm{A}$. S. Bachmeier, "Introducing the next-generation advanced baseline imager on GOES-R," Bulletin of the American Meteorological Society, vol. 86, no. 8, pp. 1079-1096, 2005.

[28] J. Li, W. W. Wolf, W. P. Menzel, W. Zhang, H. L. Huang, and T. H. Achtor, "Global soundings of the atmosphere from ATOVS measurements: the algorithm and validation," Journal of Applied Meteorology, vol. 39, no. 8, pp. 1248-1268, 2000.

[29] S.-Y. Hong and J.-O. Lim, "The WRF single-moment microphysics scheme (WSM6)," Journal of the Korean Meteorological Society, vol. 42, pp. 129-151, 2006.

[30] G. A. Grell and D. Dévényi, "A generalized approach to parameterizing convection combining ensemble and data assimilation techniques," Geophysical Research Letters, vol. 29, no. 14, article 1693, 2002.

[31] D. F. Parrish and J. C. Derber, "The National Meteorological Center's spectral statistical-interpolation analysis system," Monthly Weather Review, vol. 120, no. 8, pp. 1747-1763, 1992.

[32] M. T. Chahine, H. Aumann, L. Goldberg et al., "AIRS-team retrieval for core products and geophysical parameters, Level 
2, Algorithm Theoretical Basis Document Version 2.2," NASA, 2001.

[33] G. Masiello, C. Serio, and P. Antonelli, "Inversion for atmospheric thermodynamical parameters of IASI data in the principal components space," Quarterly Journal of the Royal Meteorological Society, vol. 138, pp. 103-117, 2012. 

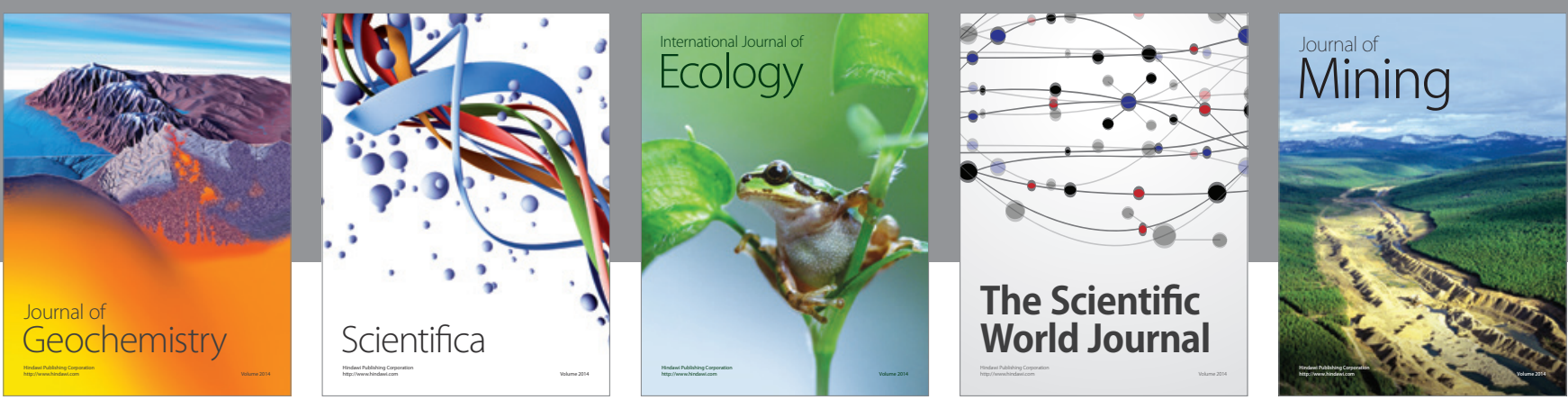

The Scientific World Journal
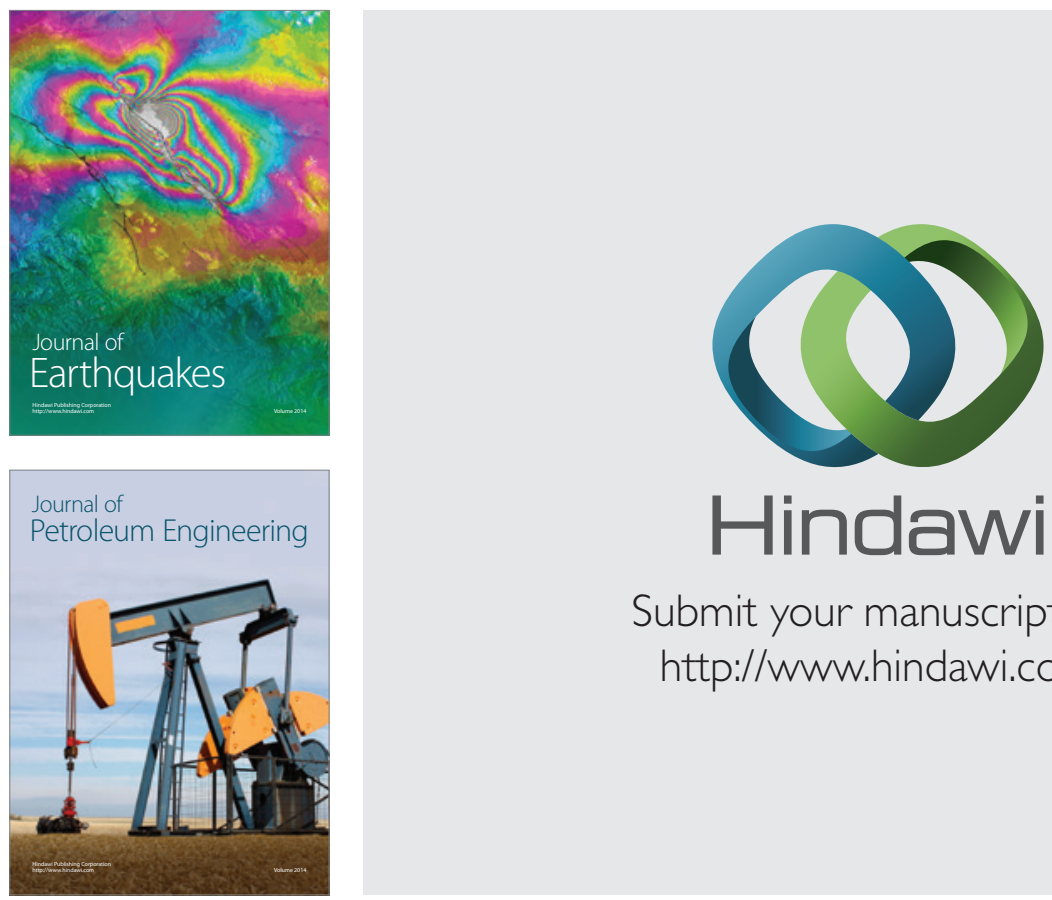

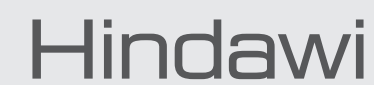

Submit your manuscripts at

http://www.hindawi.com
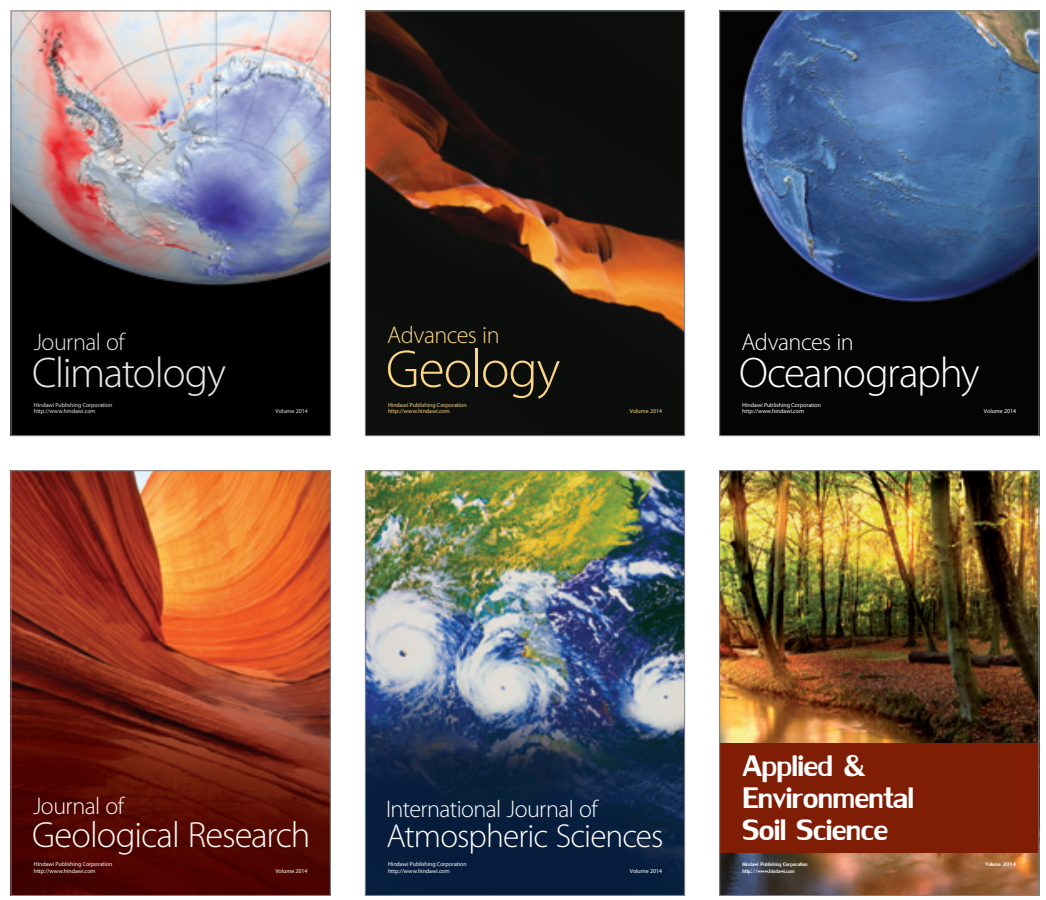
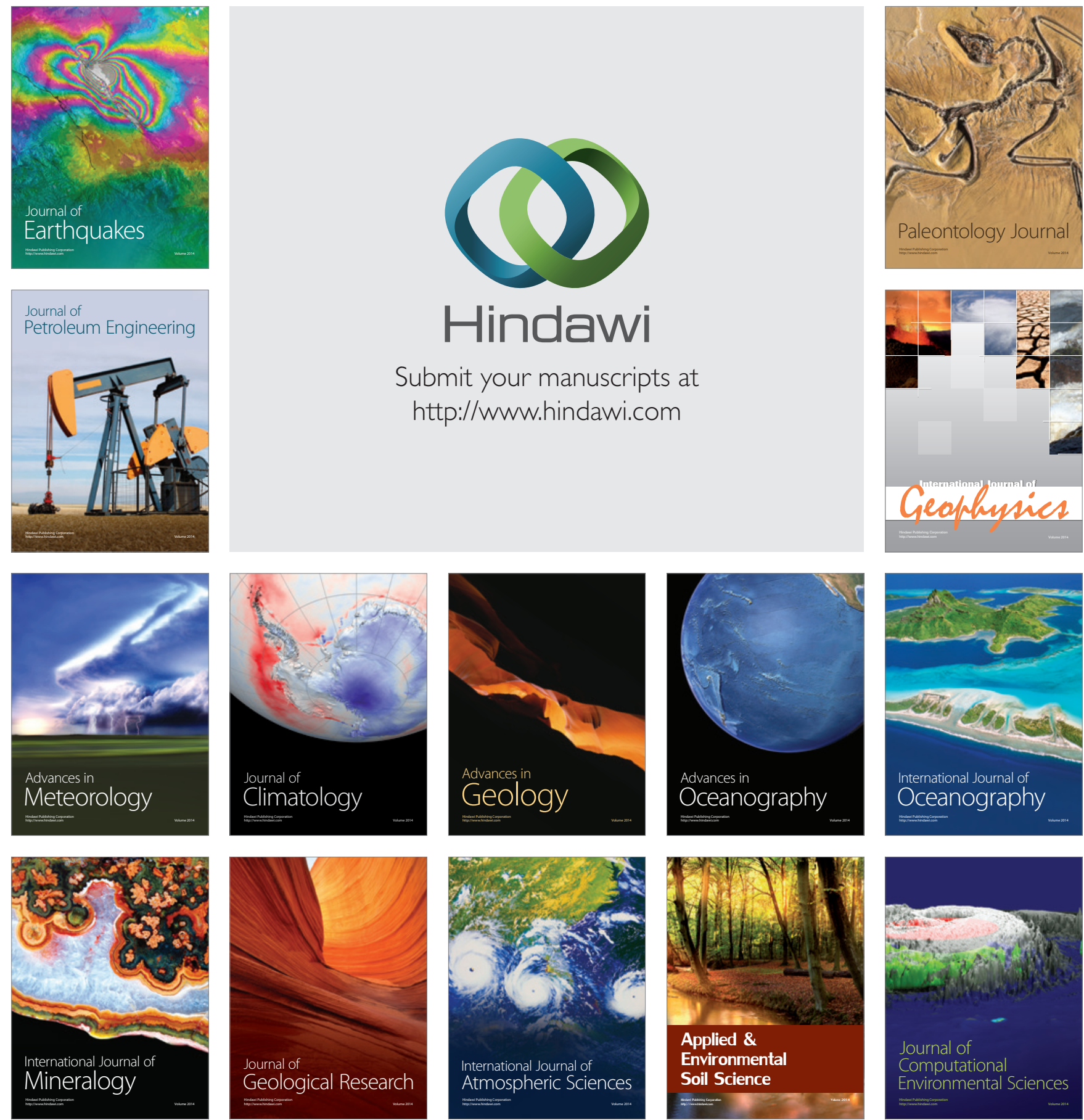